\title{
Nucleation-Elongation Dynamics of Two-Dimensional Covalent Organic Frameworks
}

\author{
Supplementary Information
}

Haoyuan Li ${ }^{a \S}$, Austin M. Evans ${ }^{b \S}$, Ioannina Castano ${ }^{b}$, Michael J. Strauss $^{b}$, William R. Dichtel $^{b *}$ and Jean-Luc Bredas ${ }^{a *}$

aSchool of Chemistry and Biochemistry, Center for Organic Photonics and Electronics (COPE), Georgia Institute of Technology, Atlanta, Georgia 30332-0400

bDepartment of Chemistry, Northwestern University, Evanston, IL 60208, USA.

Corresponding Authors

*jean-luc.bredas@chemistry.gatech.edu

*wdichtel@,northwestern.edu

\section{Table of Contents}

I. Simulation Results ....................................................................................S2

II. Materials and Instrumentation ....................................................58

III. Synthesis of COF-5 Seeds..................................................................S9

IV. Experimental in situ Scattering Details.........................................S10

V. X-Ray Scattering Control Experiments .........................................S13

VI. In Situ X-Ray Scattering Nucleation Results................................S15

VII. In Situ X-Ray Scattering Growth Results ...................................S21

VIII. In Situ X-Ray Scattering on Other Systems ..............................S25

IX. Simulated X-ray Diffraction Models ...............................................S33

X. Derivation of Eqs. (4)-(8)..........................................................................S37

XI. References ...................................................................................................S39 


\section{Simulation Results}

The parameters used in the KMC simulations have been documented in our previous work. ${ }^{1}$ In the present KMC simulations, nuclei have been identified by setting a size threshold of 50 monomer units, which we choose to be slightly larger than the value of 40 monomer units used in our previous study ${ }^{1}$ in order to ensure better identification. Figure $\mathbf{S} 1$ shows a typical nucleus production curve. For each simulation, a linear fitting is performed. The nucleation rate corresponds to the fitted slope.

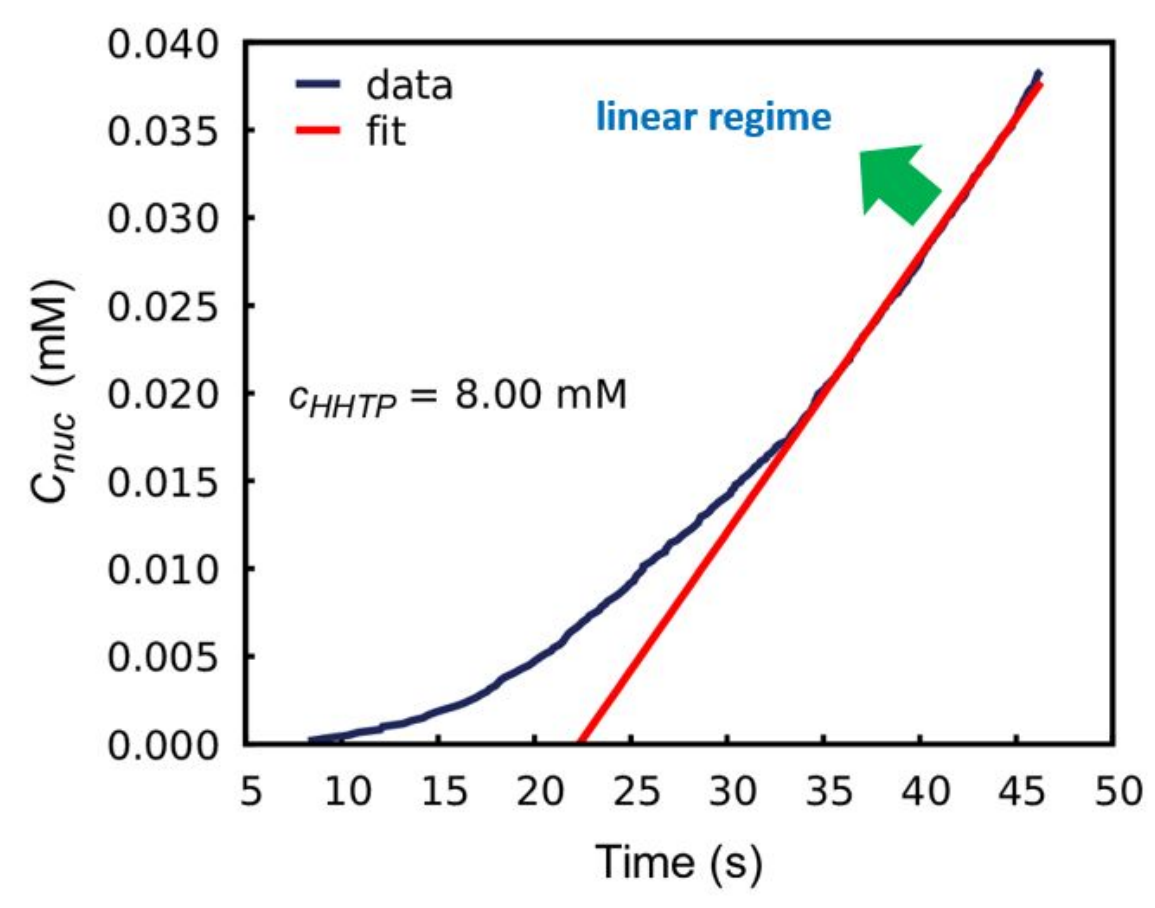

Figure S1. Representative Linear Fitting Used in the KMC Simulations: Illustration of the calculation of the nucleation rates. 


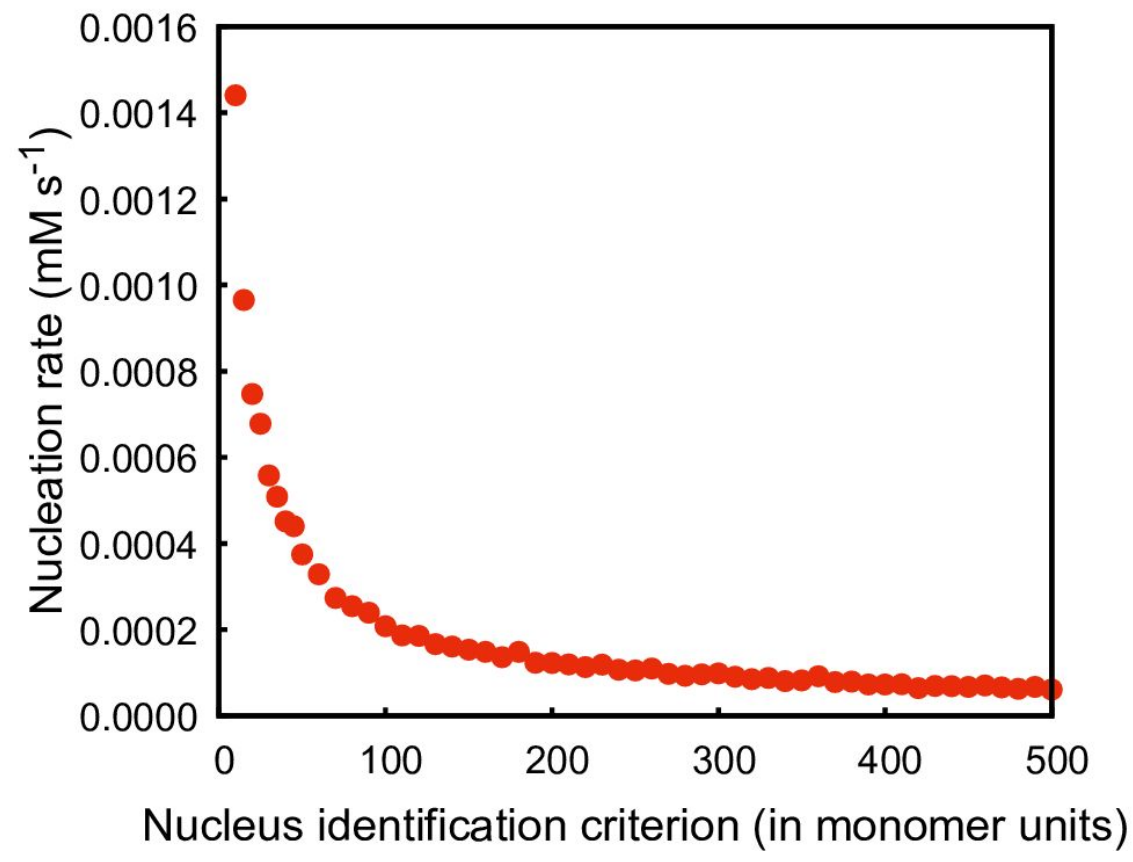

Figure S2. Impact of the size criterion in the identification of nuclei on the calculated nucleation rate.

For nucleation simulations, the total number of monomers is 120,500 . We have checked that the error coming from the finite sizes of the simulation system is not significant, as shown in Figure S3. 


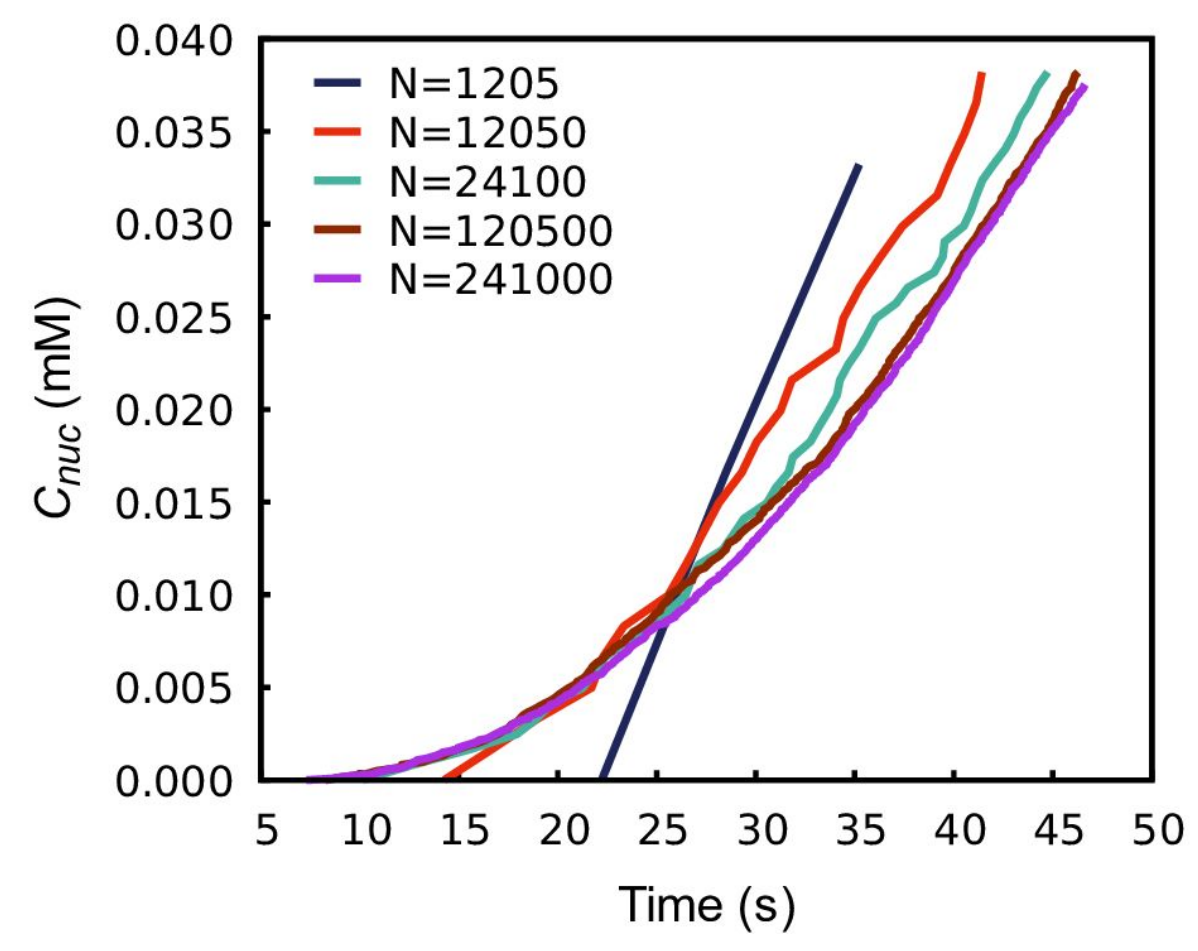

Figure S3. Simulation Results for Different System Sizes in the KMC Simulations. N is the total number of monomers. The concentrations of monomers are the same across all simulations.

The lateral expansion is found to be approximately linear with time. Figures S4 and S5 show a typical COF expansion in the course of the simulated growth and the top view of the final crystal, respectively. Linear fittings are performed on the crystal expansion results, see Figure S6. The lateral expansion rate thus corresponds to the fitted slope. 


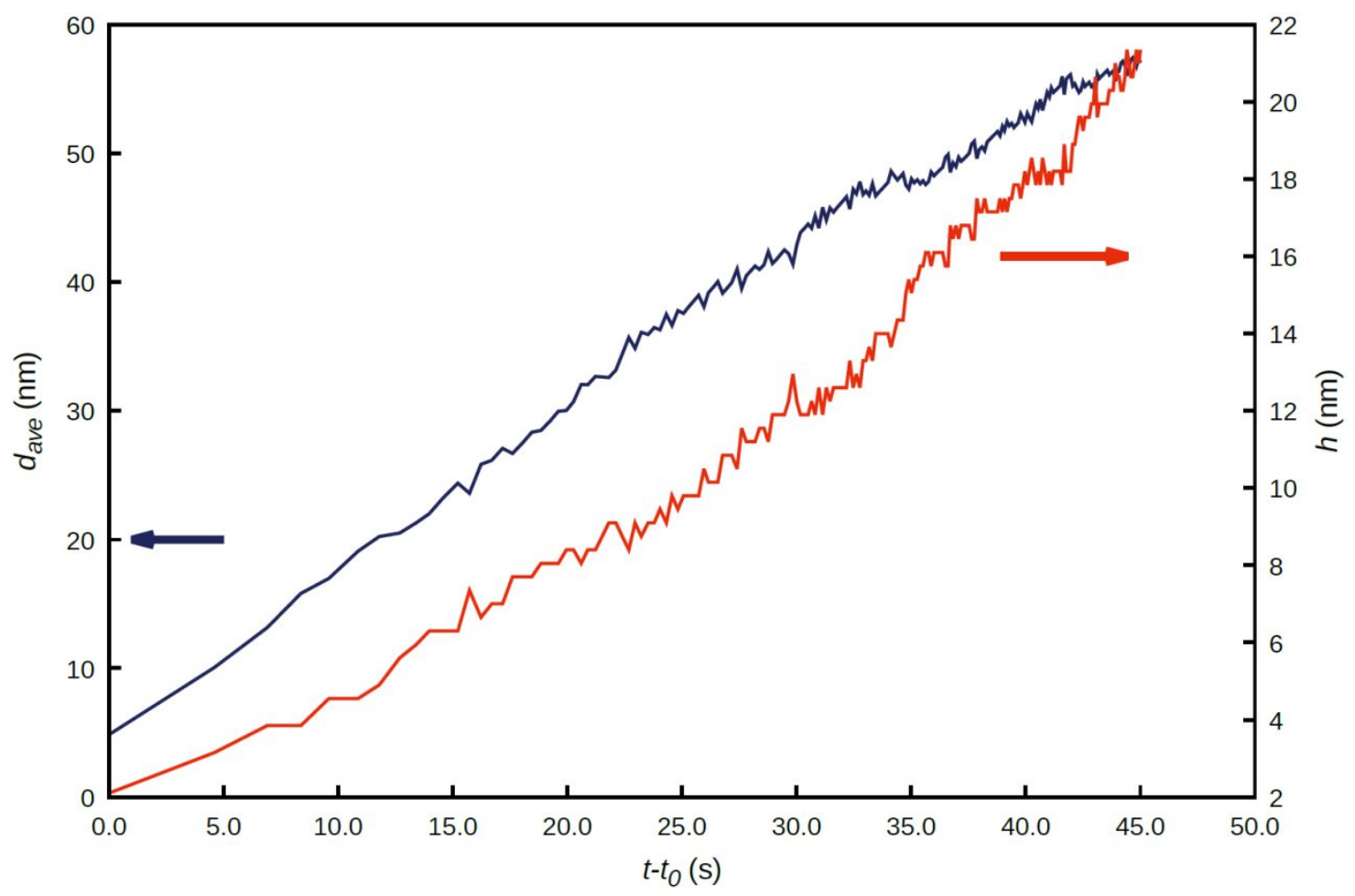

Figure S4. Example of KMC Growth Result. Illustration of the evolution of average diameter and height as a function of time. 


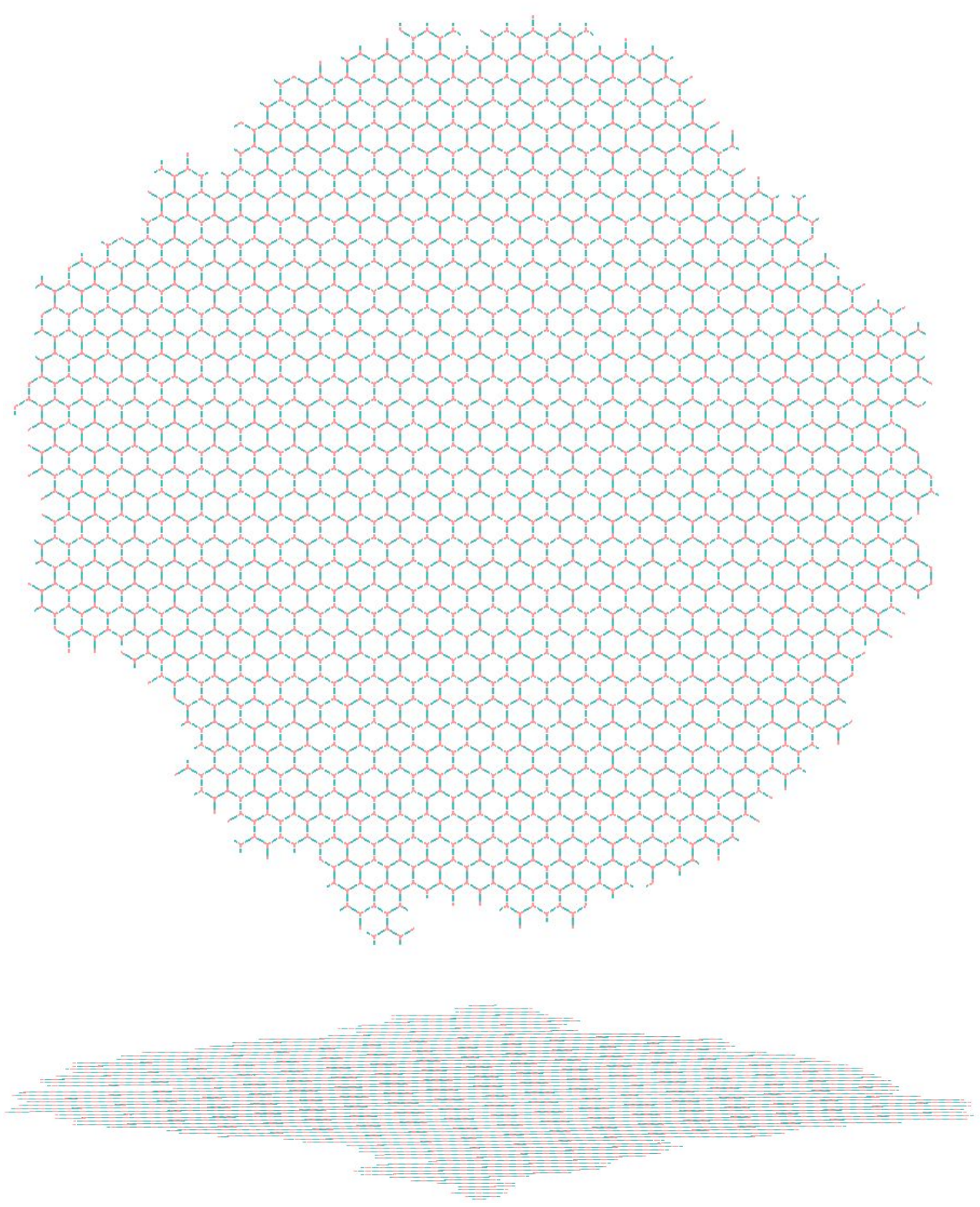

Figure S5. COF-5 particle simulation. Illustration of the final structure of the COF-5 crystal (top view and side view) from the KMC simulation. 


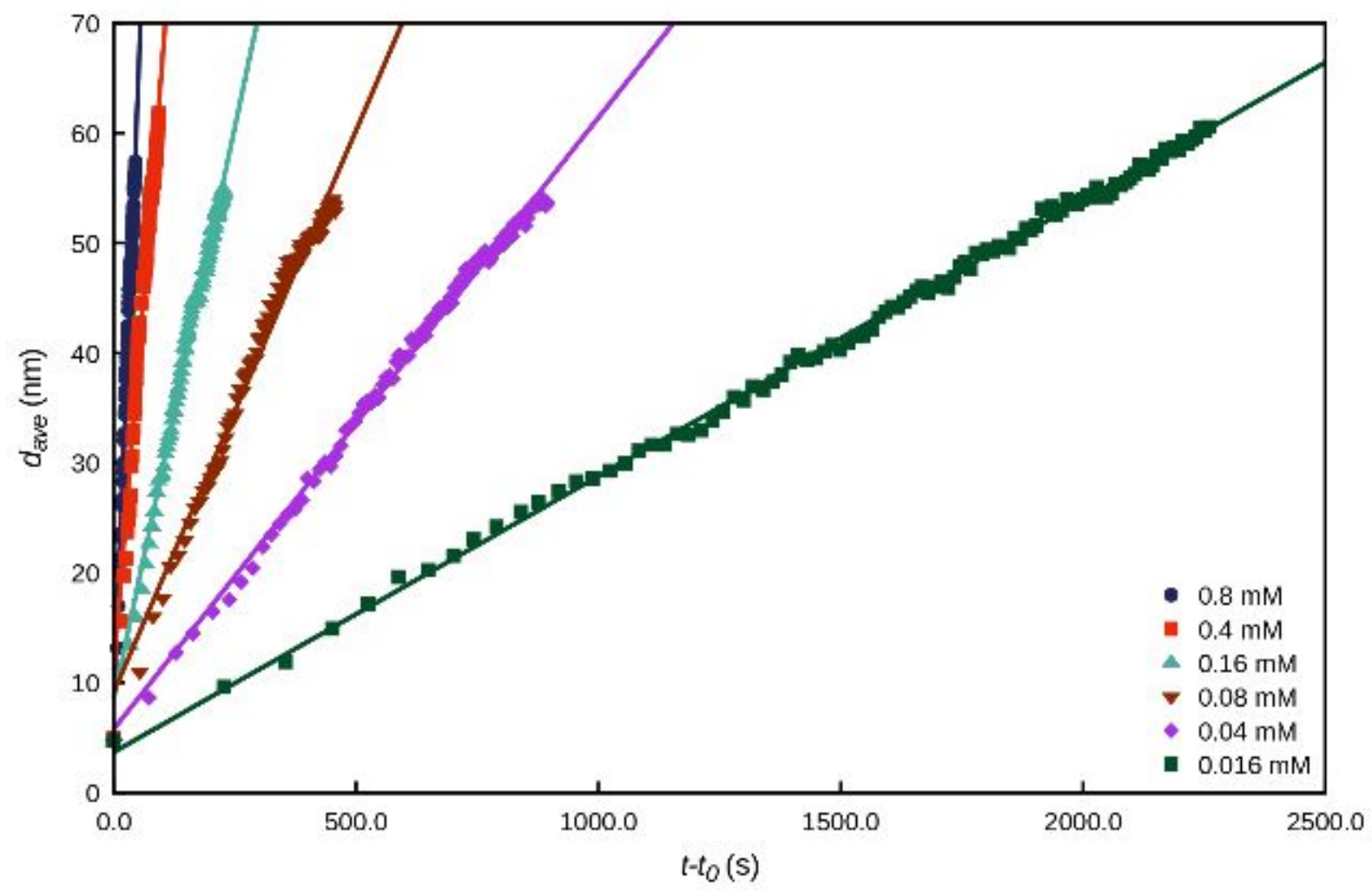

Figure S6. Fitting of the Lateral Expansion Data. Lateral expansion as a function of time and linear fittings. 


\section{Materials and Instrumentation}

\section{Materials}

Acetonitrile, 1-4 Dioxane, and Mesitylene were purchased from Fisher Scientific. Phenylbisboronic acid (PBBA) was purchased from Sigma-Aldrich and hexahydroxytriphenylene (HHTP) was purchased from TCI America. All chemicals were used without further purification.

\section{Instrumentation}

Sonication. Sonication was performed with a Branson 3510 ultrasonic cleaner with a power output of $100 \mathrm{~W}$ and a frequency of $42 \mathrm{kHz}$.

In Situ X-ray Scattering: Small/Medium/Wide-Angle X-ray Scattering (SAXS/MAXS/WAXS) data were collected simultaneously at sector 5-ID-D of the Advanced Photon Source at Argonne National Laboratory. A beam energy of $17.0 \mathrm{KeV}$ was used for these experiments. Patterns were collected with single $10 \mathrm{~s}$ frames on a series of 3 Pilatus $2 \mathrm{D}$ detectors which were then radially integrated. All samples were conducted in $1.5 \mathrm{~mm}$ borosilicate capillaries with a wall thickness of $0.01 \mathrm{~mm}$ available from Charles Supper Scientific. Patterns were assigned to be time $=0$ seconds when the heater reached its steady state temperature of $70^{\circ} \mathrm{C}$ (approximately 45 seconds). Patterns were baselined and then background subtracted from the starting time pattern to produce corrected patterns. The $<100>$ Bragg feature of COF-5 $\left(\sim 0.24 \AA^{-1}\right)$ was then integrated and plotted against the time of each pattern. Finally, the rate of increase in $<100>$ signal intensity was determined by fitting the slope of the respective $<100>$ intensity versus time for each experiment. 


\section{Synthesis of COF-5 Seeds}

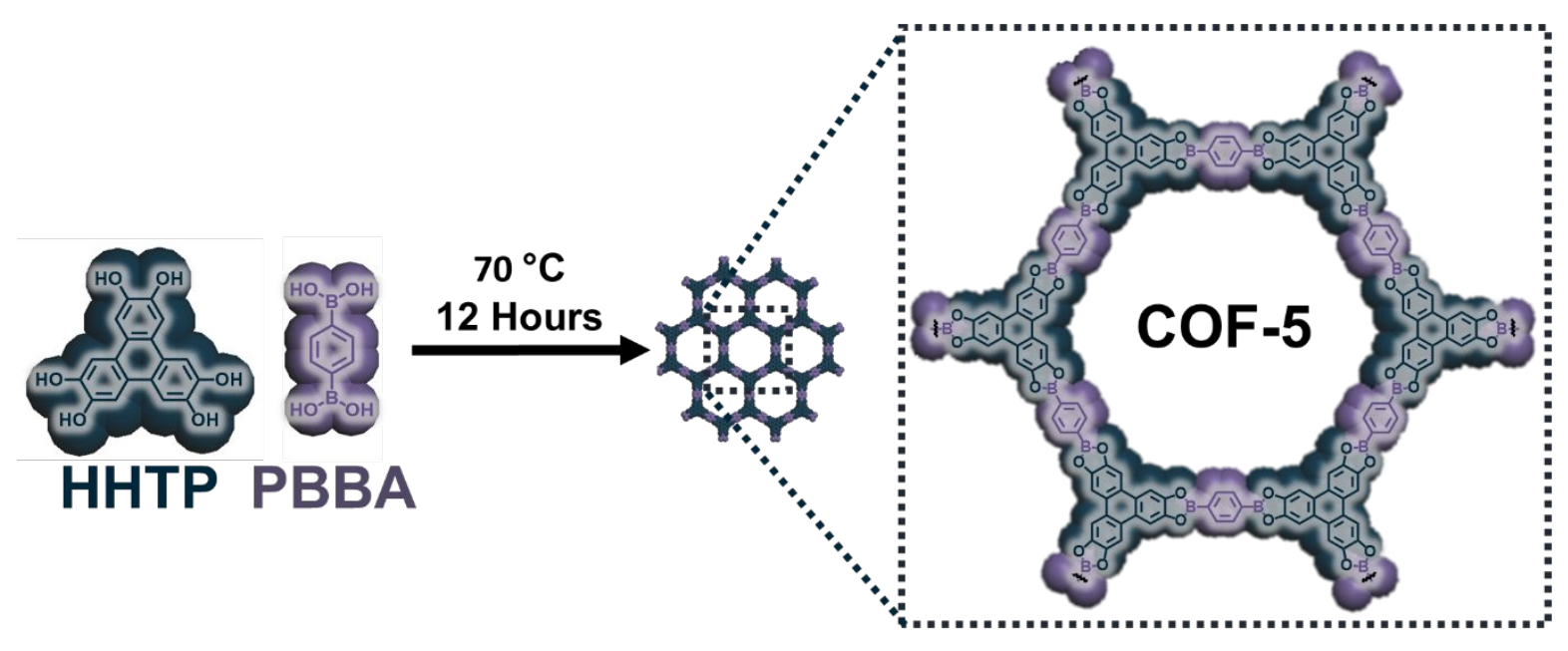

Figure S7. Synthesis Procedure of COF-5 Colloidal Seeds.

Hexahydroxytriphenylene (HHTP) and phenylbisboronicacid (PBBA) were added to a $20 \mathrm{~mL}$ mixture of $\mathrm{CH}_{3} \mathrm{CN}$ :dioxane:mesitylene (80:16:4 vol\%) at a concentration of $2 \mathrm{mM}$ HHTP and 3 mM PBBA. This solution was sonicated until full dissolution of the monomers was observed. This solution was then heated in a scintillation vial overnight at $70{ }^{\circ} \mathrm{C}$ after which time the solution became opaque indicating the successful synthesis of COF-5 seed particles. These seeds were then used as prepared for COF growth experiments detailed below. This method is explained in further detail in Ref. ${ }^{2}$ 


\section{Experimental in situ Scattering Details}
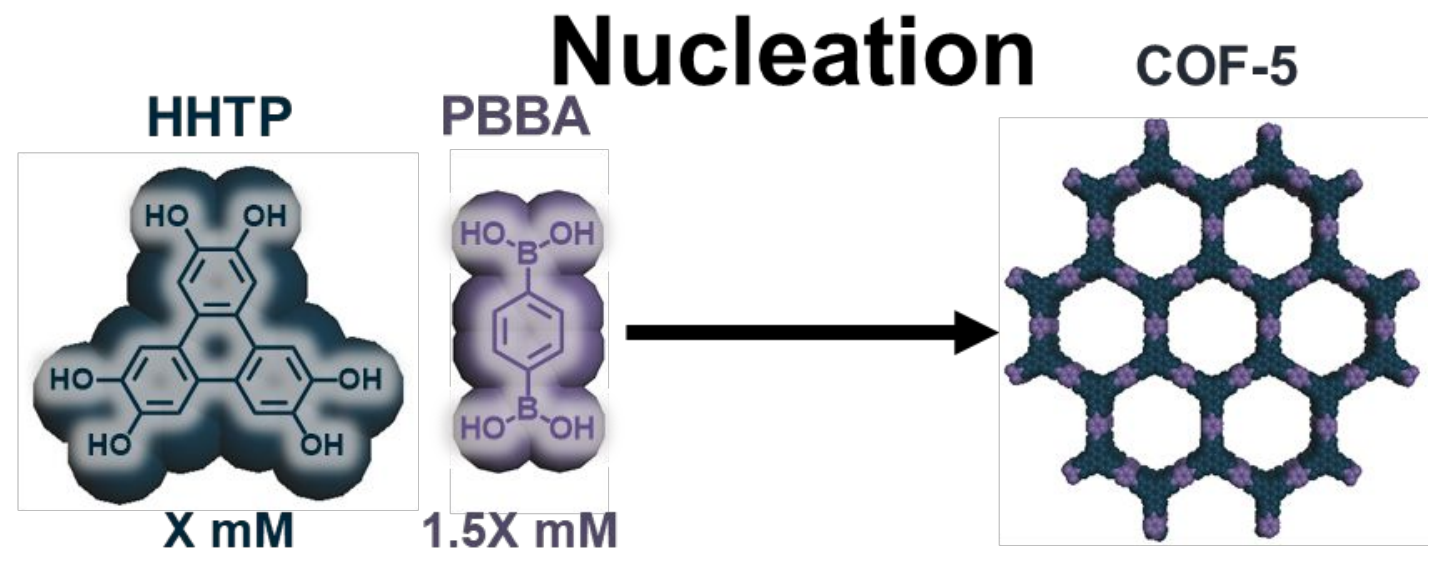

Figure S8. Nucleation Experiment. Schematic of how the nucleation experiments were conducted.

Nucleation Experiment: Hexahydroxytriphenylene (HHTP) and phenylbisboronicacid (PBBA) were added to a $20 \mathrm{~mL}$ mixture of $\mathrm{CH}_{3} \mathrm{CN}$ :dioxane:mesitylene (80:16:4 vol\%) in a 1:1.5 molar ratio at a concentration of double that specified in the experiment. For example, if the nucleation experiment was conducted at $2 \mathrm{mM}$, an HHTP solution of $4 \mathrm{mM}$ and a PBBA solution of $6 \mathrm{mM}$ would have been made independently. This is to prevent reaction before the mixing of the two monomers. These solutions were sonicated until full solvation of the monomers. The two monomer solutions were then mixed in a dram vial in a 1:1 volume ratio (final volume $1 \mathrm{~mL}$ ) and were then diluted as specified to produce the specified monomer concentration. These solutions were then quickly shaken to ensure mixing. Approximately $100 \mu \mathrm{L}$ of these solutions were then added to a borosilicate capillary and sealed using capillary sealing putty. These capillaries were immediately installed onto a multicapillary heating stage available at Sector 5-ID-D of the Advanced Photon 
Source (APS) Argonne National Lab (ANL). This stage was then heated to $70^{\circ} \mathrm{C}(<1$ min heating time) and allowed to react while continuous SAXS/MAXS/WAXS patterns were taken.

\section{Growth}

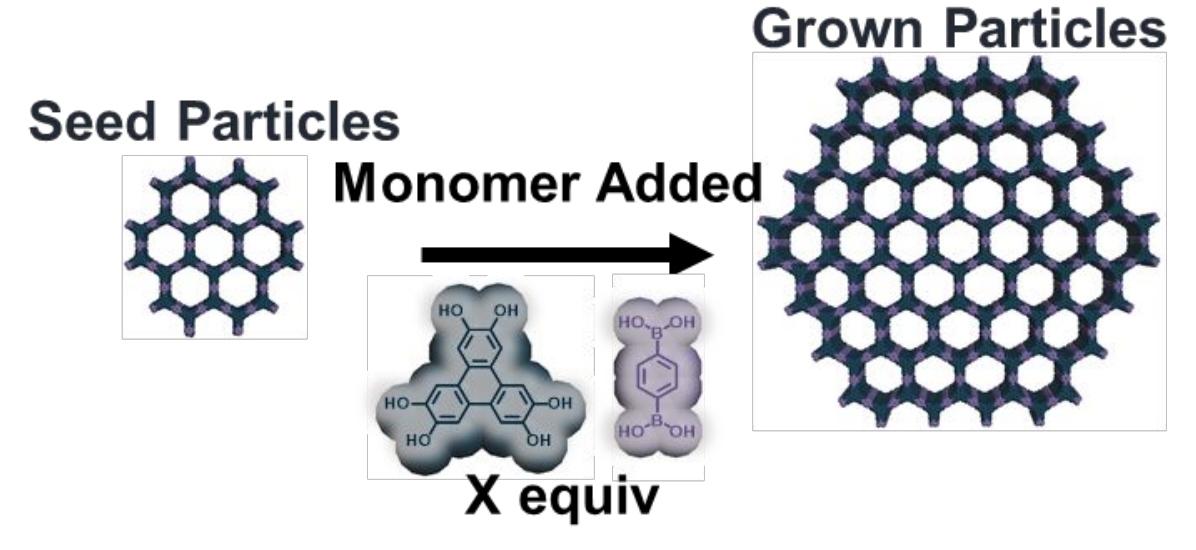

Figure S9. Growth Experiment. Schematic of how the growth experiments were conducted.

Growth Experiment: Hexahydroxytriphenylene (HHTP) and phenylbisboronicacid (PBBA) were added to a separate $20 \mathrm{~mL}$ mixture of $\mathrm{CH}_{3} \mathrm{CN}$ :dioxane:mesitylene (80:16:4 vol\%) at a concentration of $1 \mathrm{mM}$ HHTP and $1.5 \mathrm{mM}$ PBBA. These solutions were sonicated until full solvation of the monomers. The two monomer solutions were then mixed in a dram vial in a 1:1 volume ratio (final volume $2 \mathrm{~mL}$ ) and quickly shaken. Dilutions were then performed in dram vials to produce different concentrations of monomer solutions with the same volume. The highest monomer concentration $(0.48 \mathrm{mM})$ was chosen to temporally resolve nucleation and growth. The same amount of COF-5 seeds (COF-5 seeds diluted by a factor of 20 , resulting in particle solution containing $0.1 \mathrm{mM}$ HHTP and $0.15 \mathrm{mM}$ PBBA) were then added to all reactions to keep the density of seed particles identical. To ensure that Ostwald ripening or depolymerization were not relevant processes under these conditions, we also diluted our COF-5 seed particles and performed the 
experiment without the presence of additional monomers. Precise amounts of the dilution experiment are recorded below.

\begin{tabular}{cccc}
\hline $\begin{array}{c}\text { Seed Stock } \\
(\mathbf{m L})\end{array}$ & $\begin{array}{c}\text { Blank Solvent } \\
(\mathbf{m L} \mathbf{)}\end{array}$ & $\begin{array}{c}\text { Monomer Amount } \\
(\mathbf{m L})\end{array}$ & $\begin{array}{c}\text { Resultant Monomer } \\
\text { Concentration }(\mathbf{m M})\end{array}$ \\
\hline 0.1 & 0 & 1.9 & 0.48 \\
0.1 & 0.4 & 1.5 & 0.38 \\
0.1 & 0.6 & 1.3 & 0.33 \\
0.1 & 0.8 & 1.1 & 0.28 \\
0.1 & 1 & 0.9 & 0.23 \\
0.1 & 1.2 & 0.7 & 0.18 \\
0.1 & 1.9 & 0 & 0
\end{tabular}

Table S1. Dilution Calculations. Calculations of how dilutions were conducted for COF-5 growth experiments.

Approximately $100 \mu \mathrm{L}$ of these solutions were then added to a borosilicate capillary and sealed using capillary sealing putty. These capillaries were immediately installed onto a multicapillary heating stage available at Sector 5-ID-D of the Advanced Photon Source (APS) Argonne National Lab (ANL). This stage was then heated to $70{ }^{\circ} \mathrm{C}$ and allowed to react while continuous SAXS/MAXS/WAXS patterns were taken. 


\section{V.X-Ray Scattering Control Experiments}
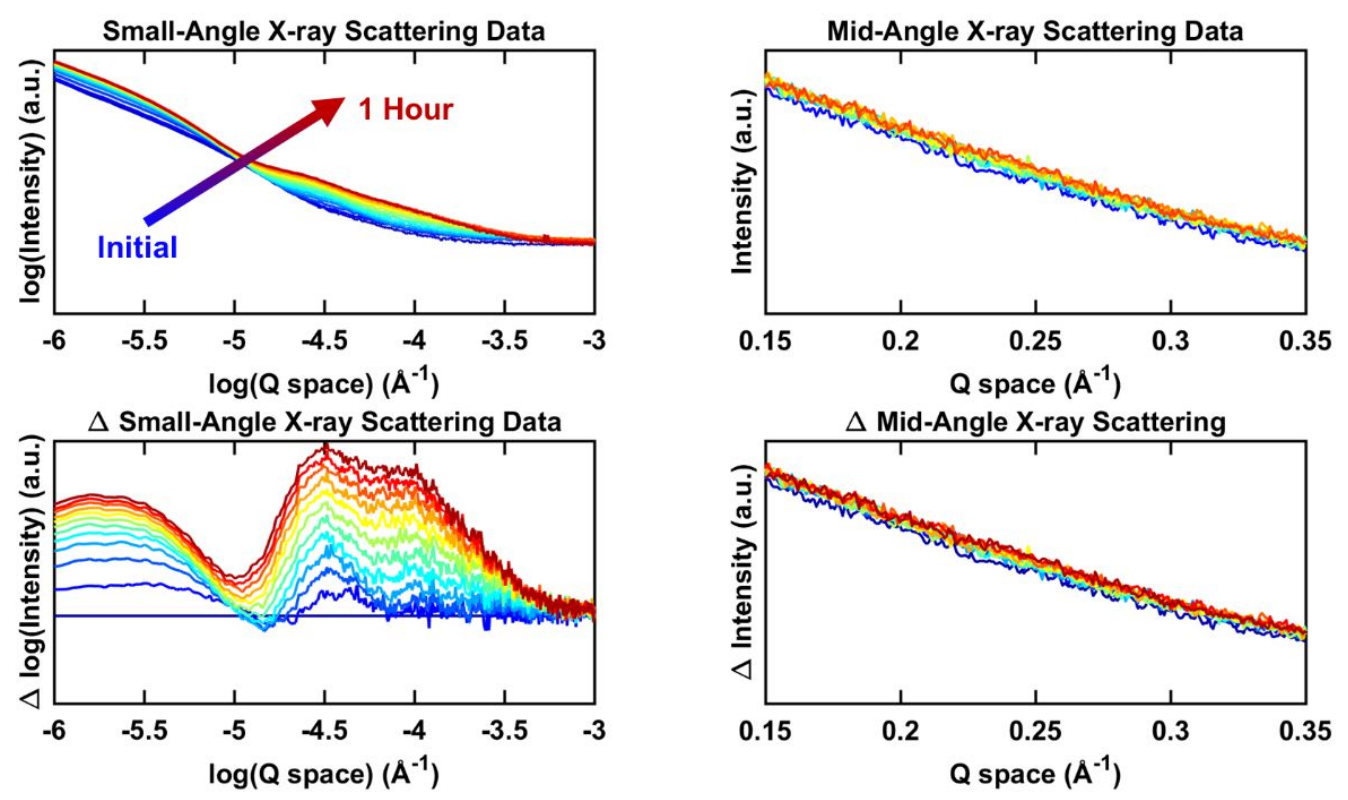

Figure S10. Nucleation Control. In situ X-ray scattering of $0.7 \mathrm{mM}$ HHTP $1.1 \mathrm{mM}$ PBBA solution over the course of 1 hour.

The background subtracted MAXS data shown in the bottom right corner demonstrates that under conditions where monomer concentration is sufficiently small results in no $<100>$ diffraction feature and thus no substantial COF nucleation on the time-scales studied here. The background subtracted SAXS data shown in the bottom left corner shows that species of small sizes are formed over the course of this reaction. This validates previous understanding of COF nucleation where small oligomers are formed but never "nucleate" unless they reach a critical concentration. 

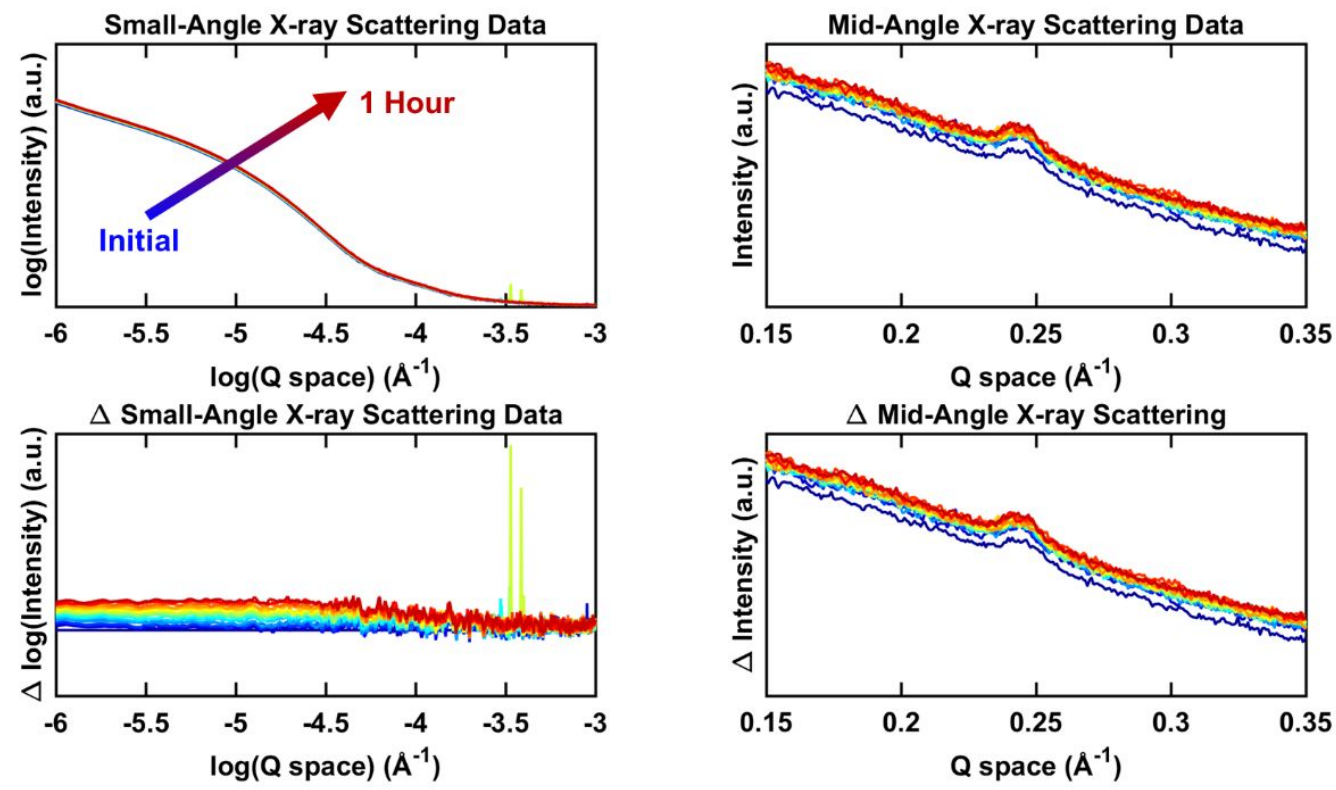

Figure S11. Growth Control. In situ X-ray scattering of COF-5 seeds heated in the presence of no additional monomer.

The MAXS data shown in the top right corner demonstrates that under conditions where no additional monomer is added no discernable difference in the $<100>$ diffraction feature is noted. The background subtracted SAXS data shown in the bottom left corner shows that there is no discernable size change of the existing nucleation after the course of one hour. These experiments suggest that the increase in intensity seen under monomer addition conditions are due to growth and not processes such as Ostwald ripening. This is consistent with observations made previously. ${ }^{2}$ 


\section{In Situ X-Ray Scattering Nucleation Results}

All experiments were conducted over the course of approximately one hour. Conditions of each experiment are given in their respective figure headings. In these figures $[$ Monomer $]=[\mathrm{HHTP}]=$ $2 / 3^{*}[\mathrm{PBBA}]$ is the concentration of monomers present at the beginning of the experiment.
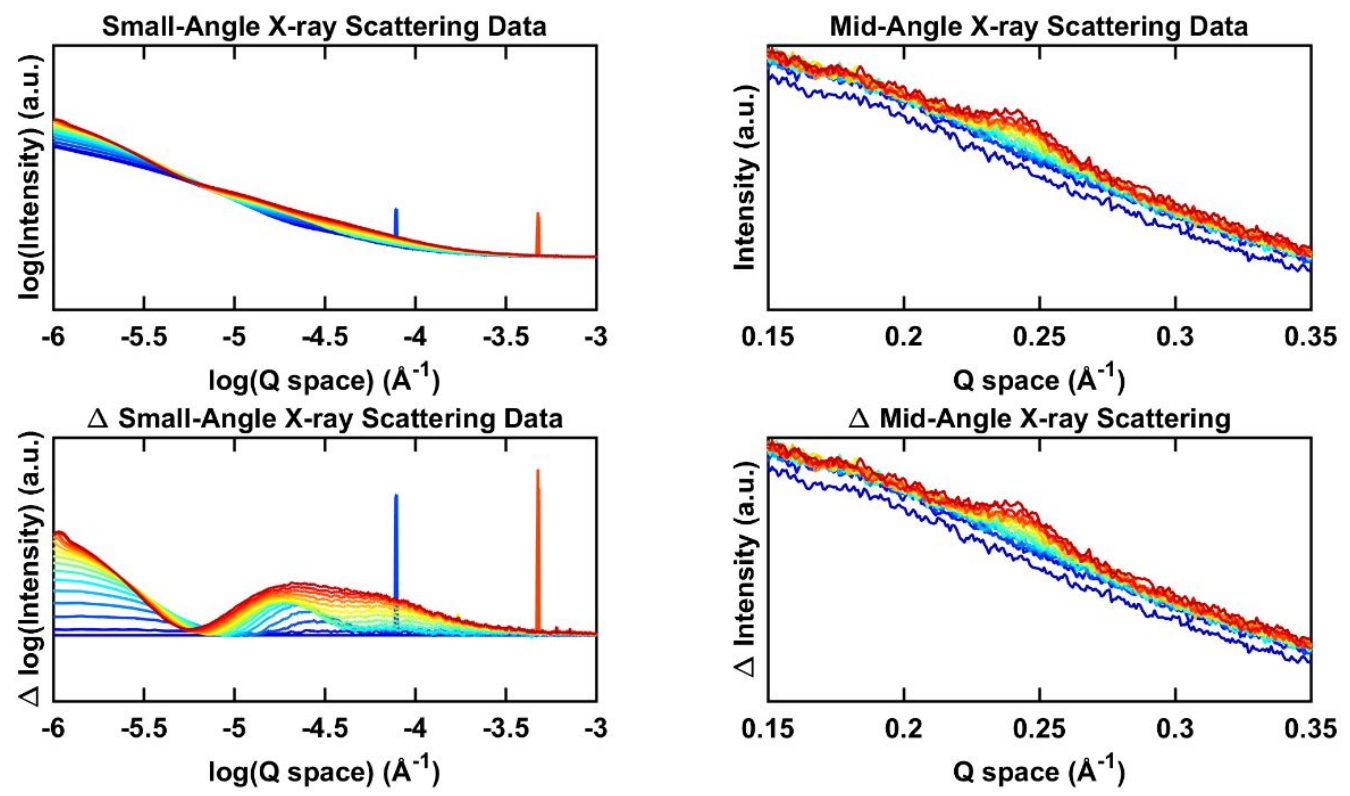

Figure S12. Nucleation Experiment [Monomer] = $0.9 \mathrm{mM}$ (measurement 1). 

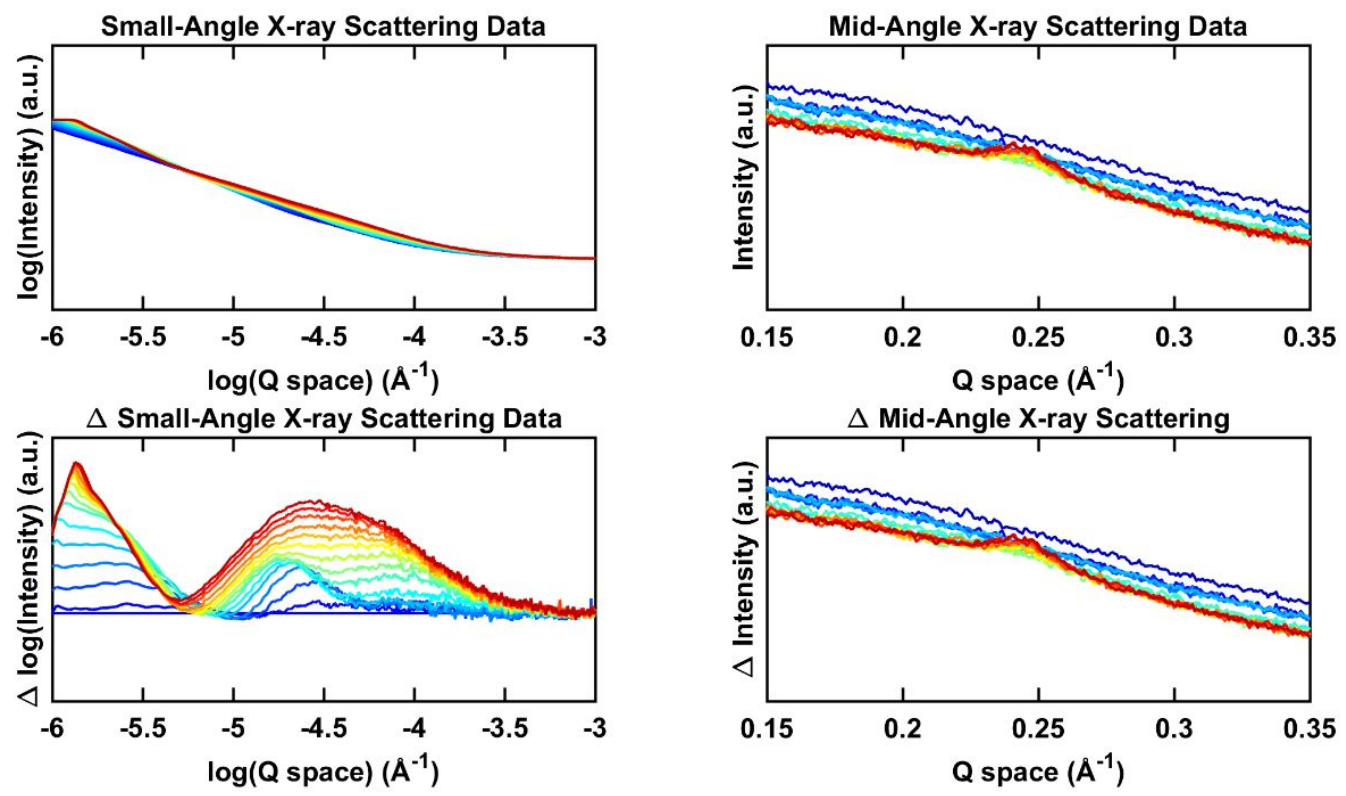

Figure S13. Nucleation Experiment [Monomer] = $0.9 \mathrm{mM}$ (measurement 2).
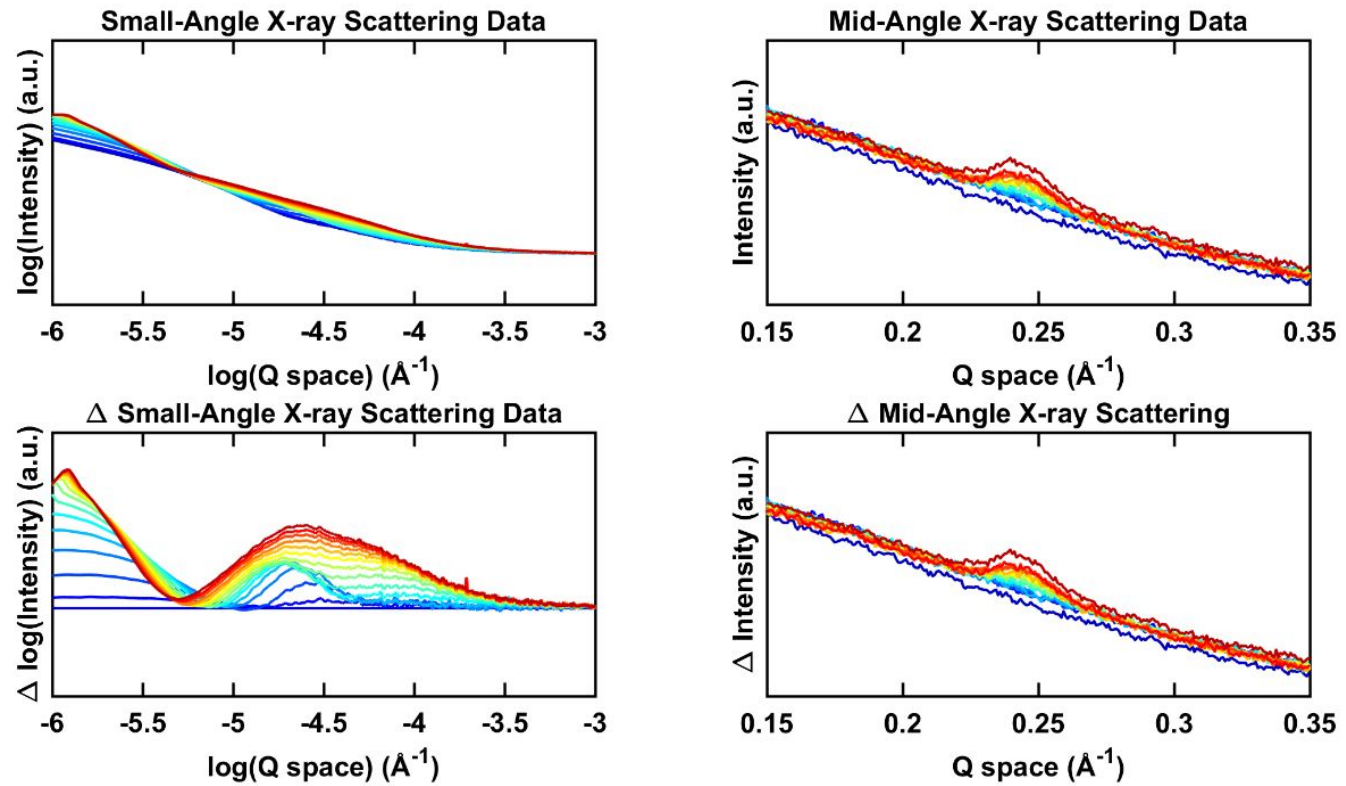

Figure S14. Nucleation Experiment [Monomer] = 1.1 mM (measurement 1). 

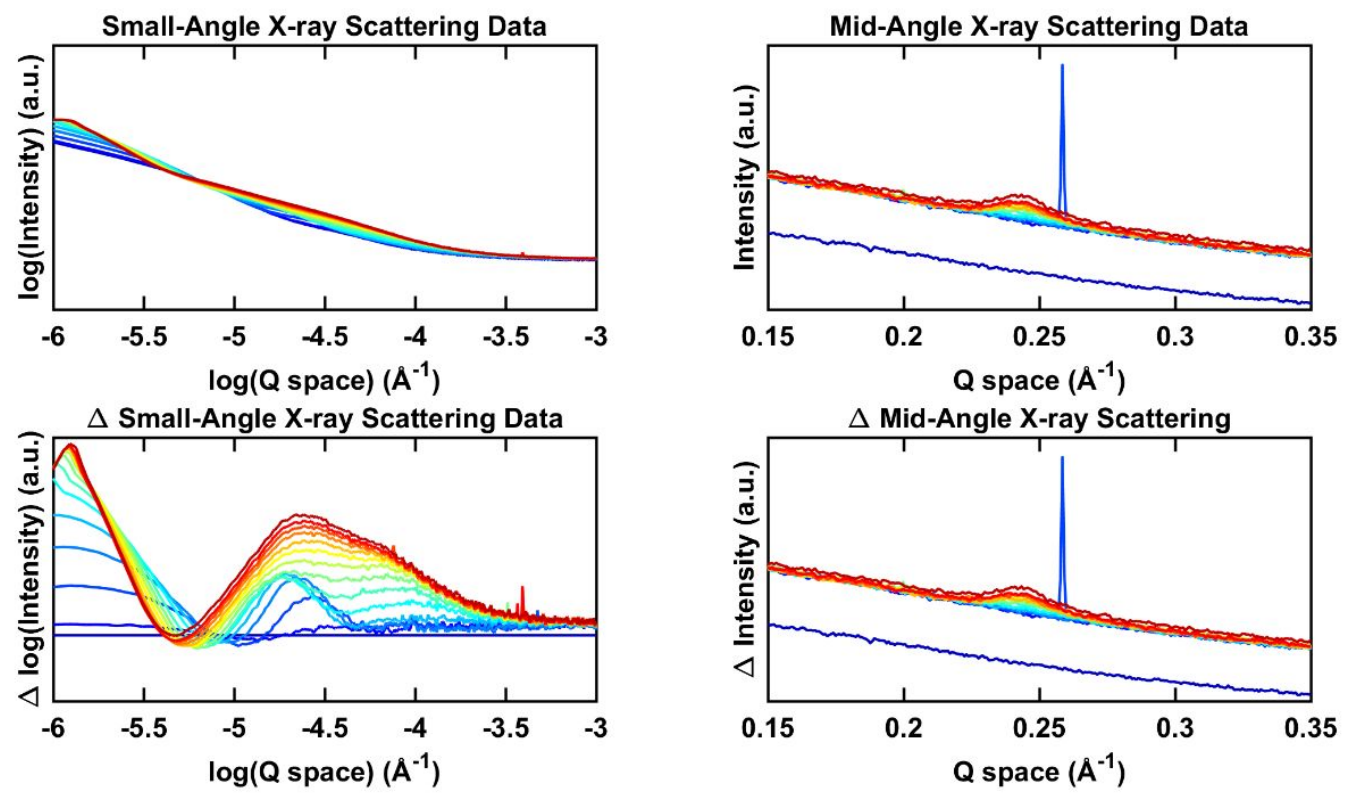

Figure S15. Nucleation Experiment [Monomer] = 1.1 mM (measurement 2).
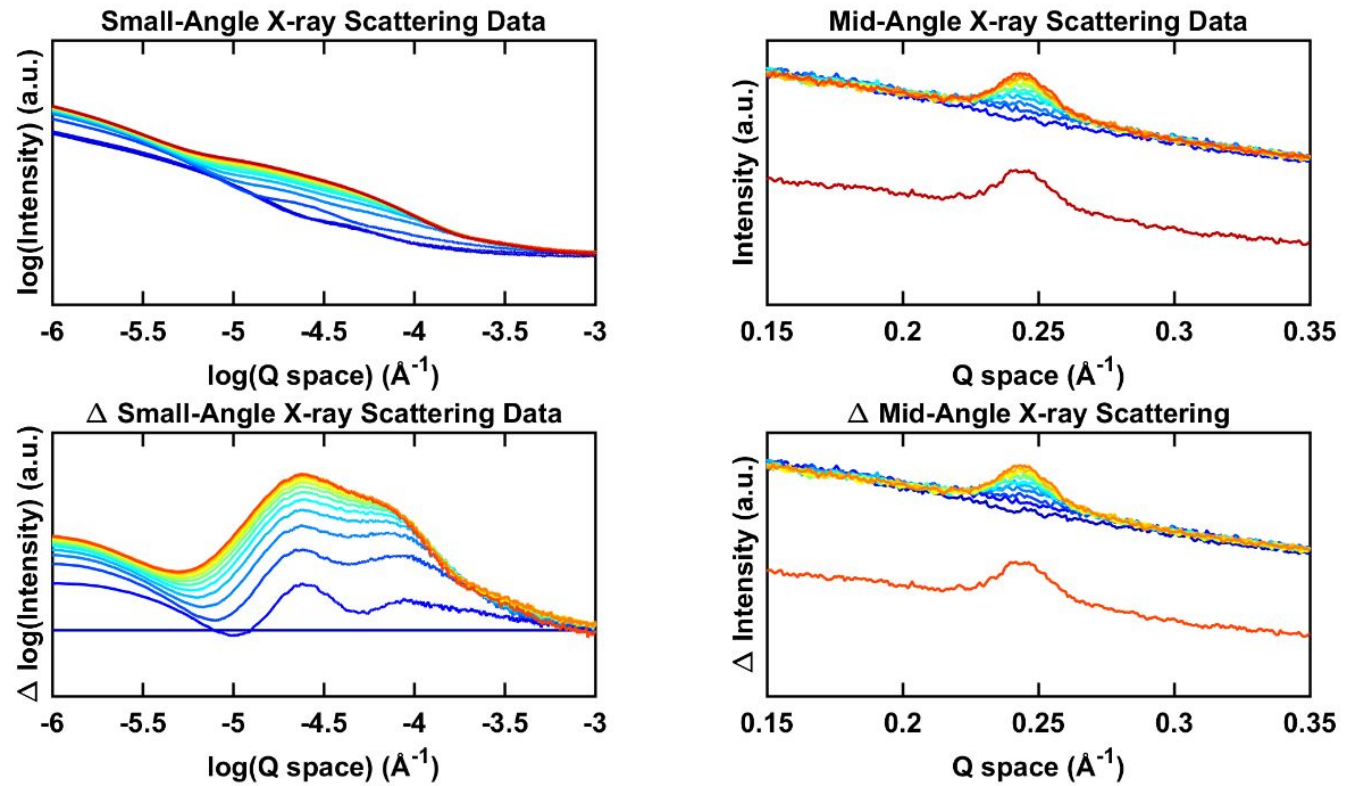

Figure S16. Nucleation Experiment [Monomer] = $1.3 \mathrm{mM}$ (measurement 1). 

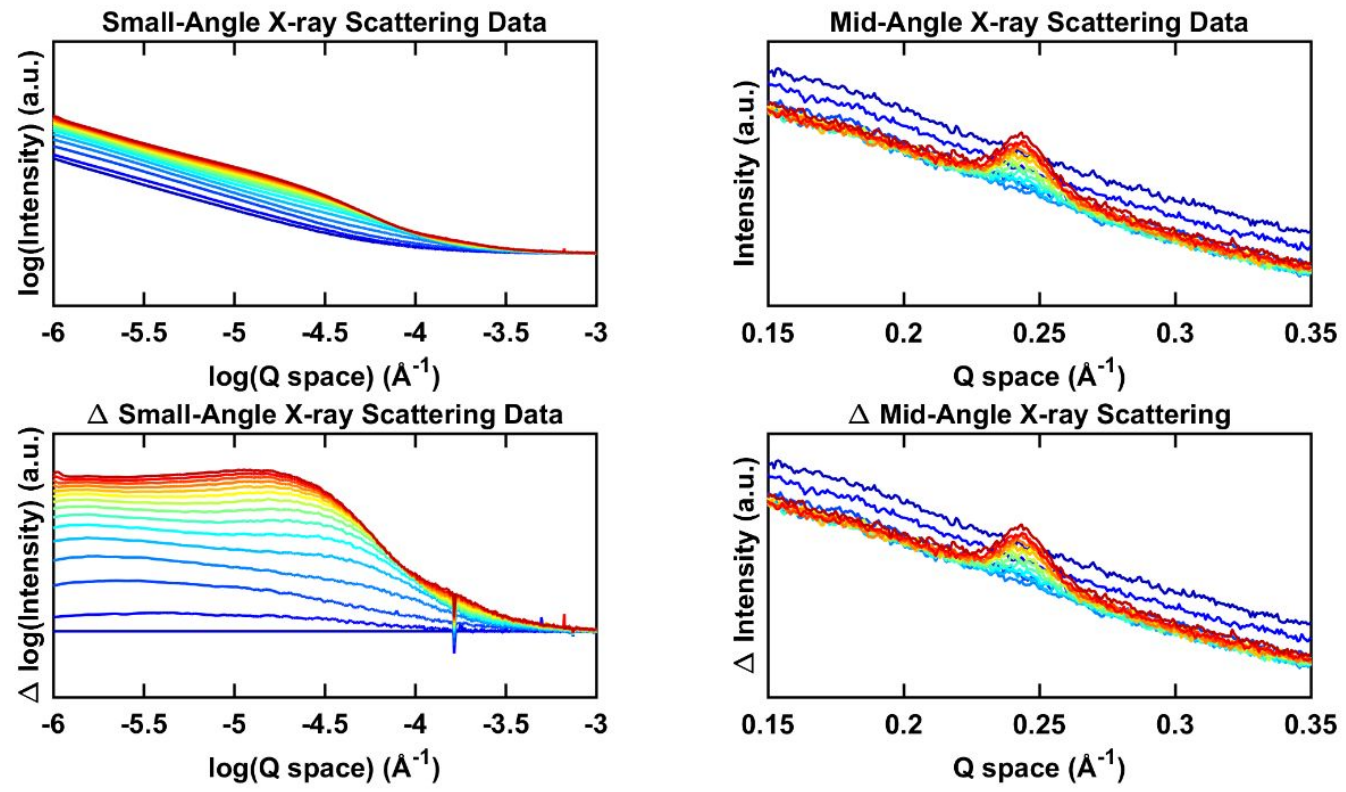

Figure S17. Nucleation Experiment [Monomer] = $1.3 \mathrm{mM}$ (measurement 2).
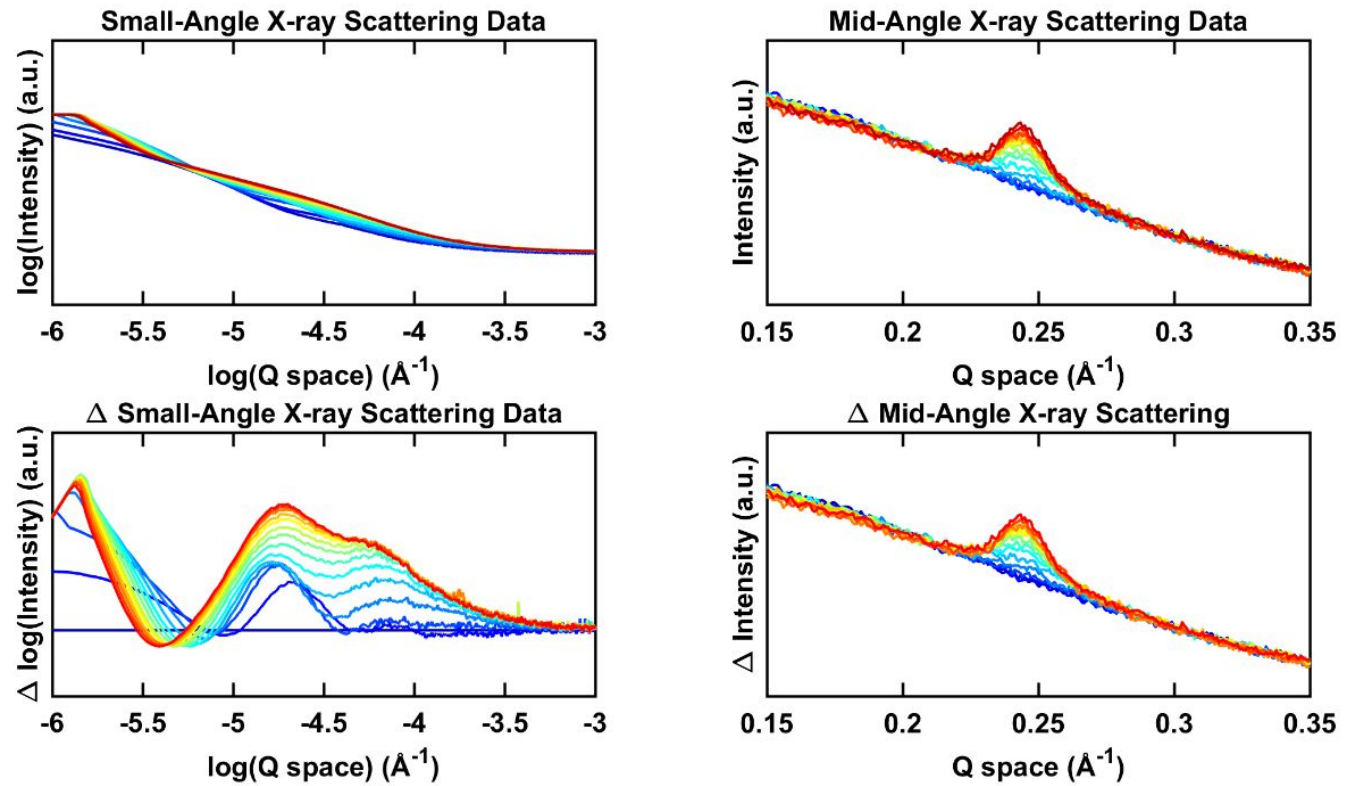

Figure S18. Nucleation Experiment [Monomer] = 1.5 mM. 

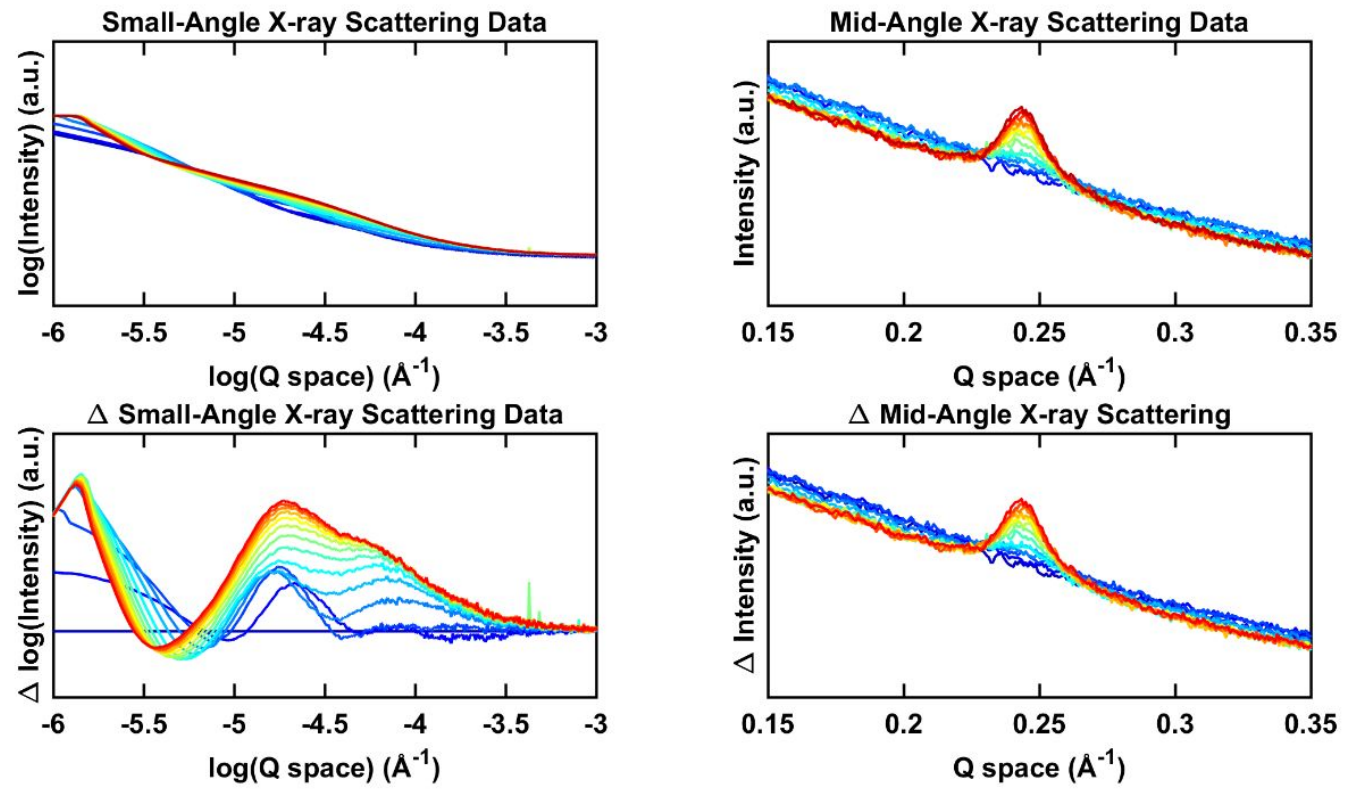

Figure S19. Nucleation Experiment [Monomer] $=1.8 \mathrm{mM}$.
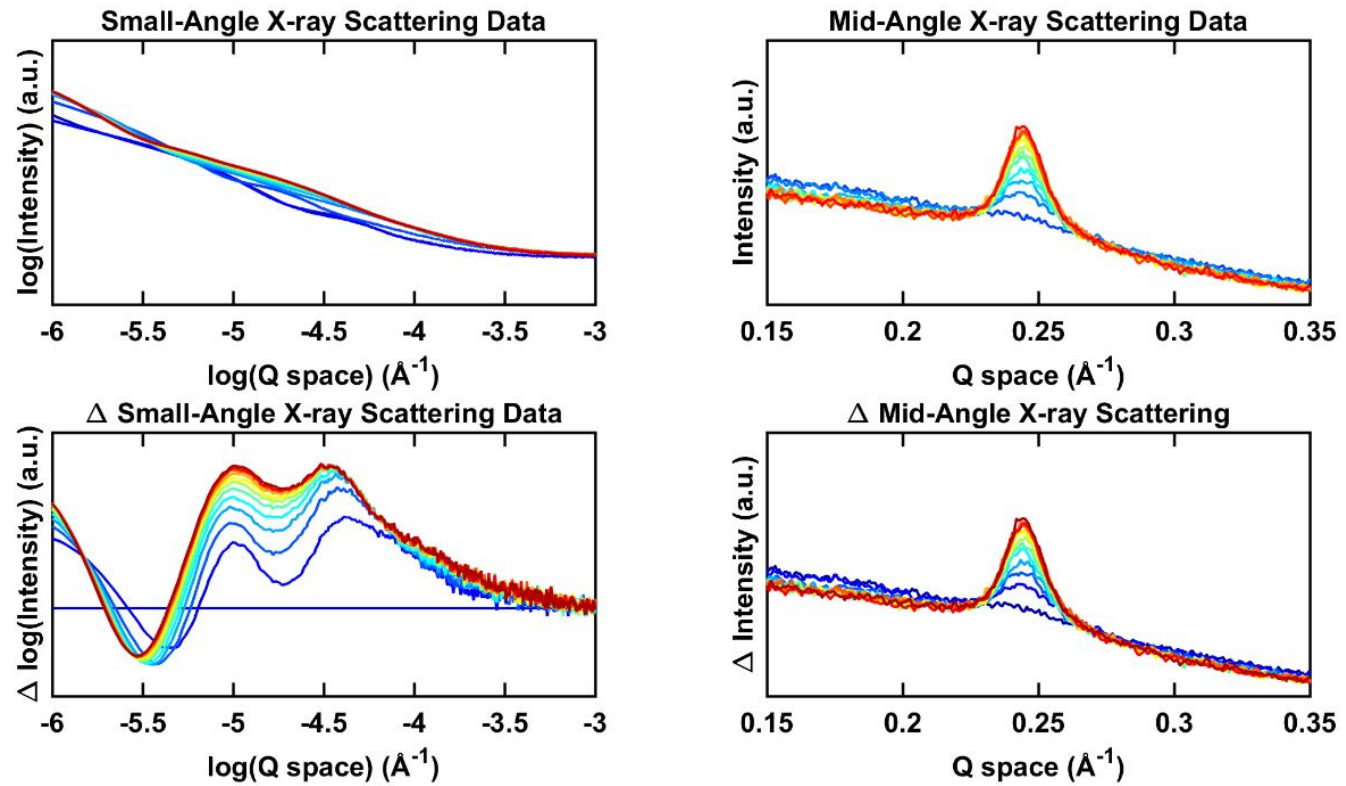

Figure S20. Nucleation Experiment [Monomer] $=2.3 \mathrm{mM}$. 

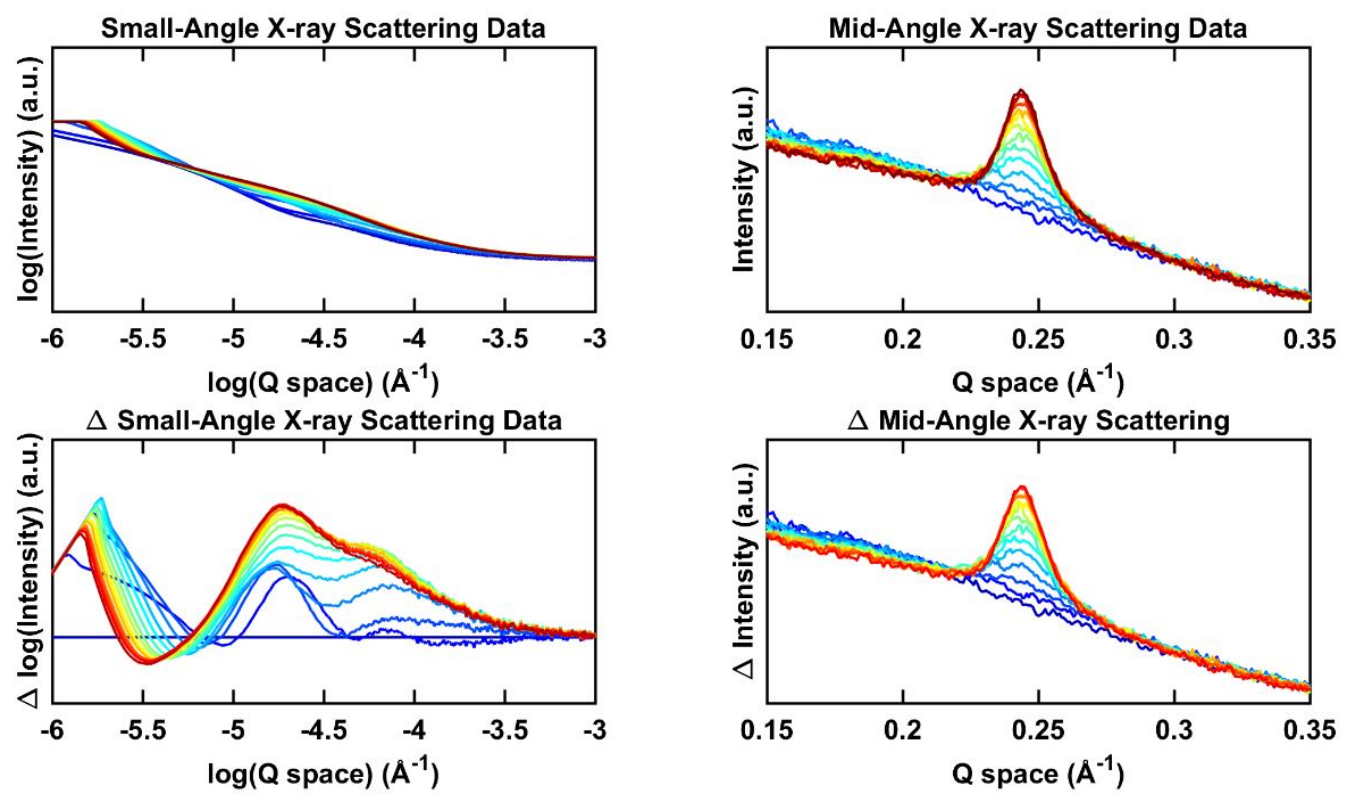

Figure S21. Nucleation Experiment [Monomer] = $3.0 \mathrm{mM}$. 


\section{In Situ X-Ray Scattering Growth Results}

All experiments were conducted over the course of approximately one hour. Conditions of each experiment are given in their respective figure headings. In these figures $[$ Monomer $]=[\mathrm{HHTP}]=$ $2 / 3 *[\mathrm{PBBA}]$ is related to the amount of monomer added to a consistent amount and concentration of preexisting COF-5 seeds.
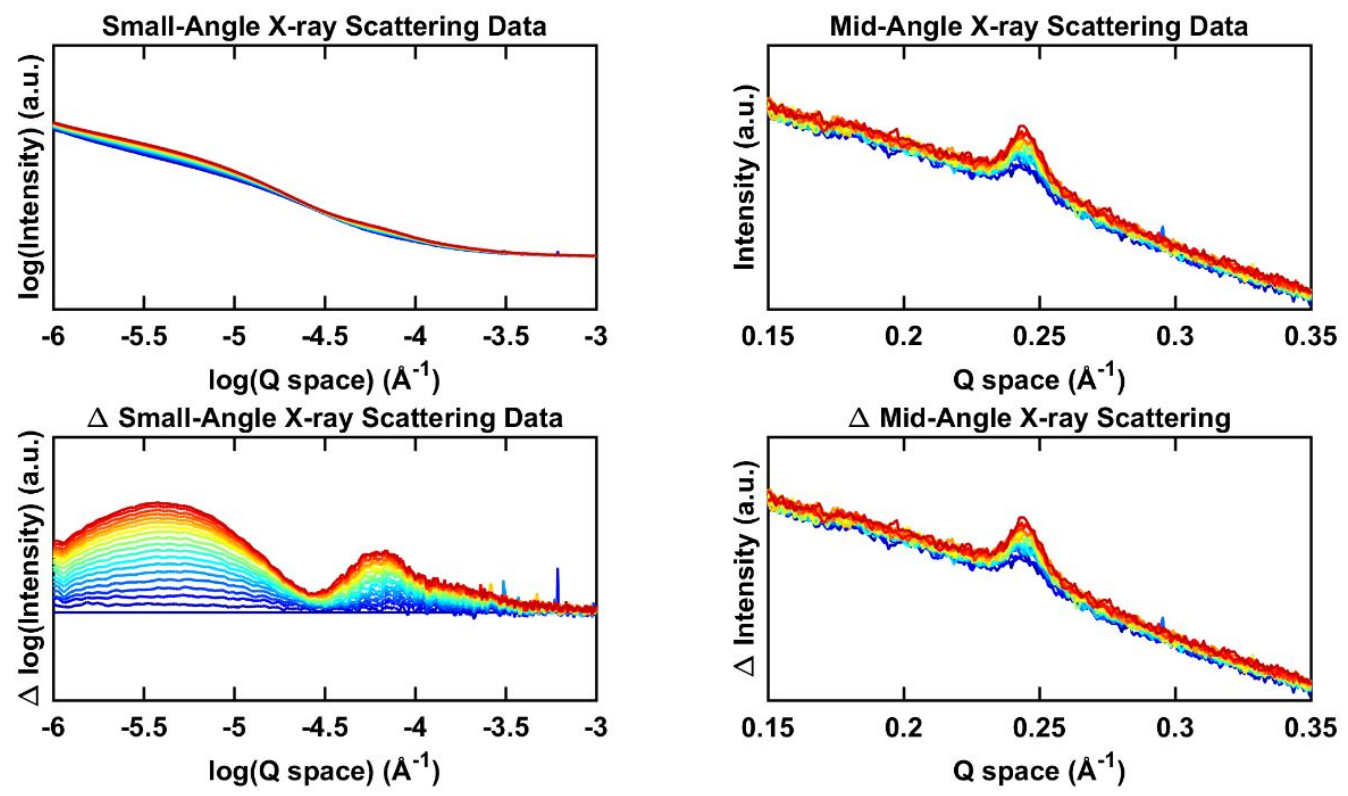

Figure S22. Growth Experiment [Monomer] = $0.18 \mathrm{mM}$. 

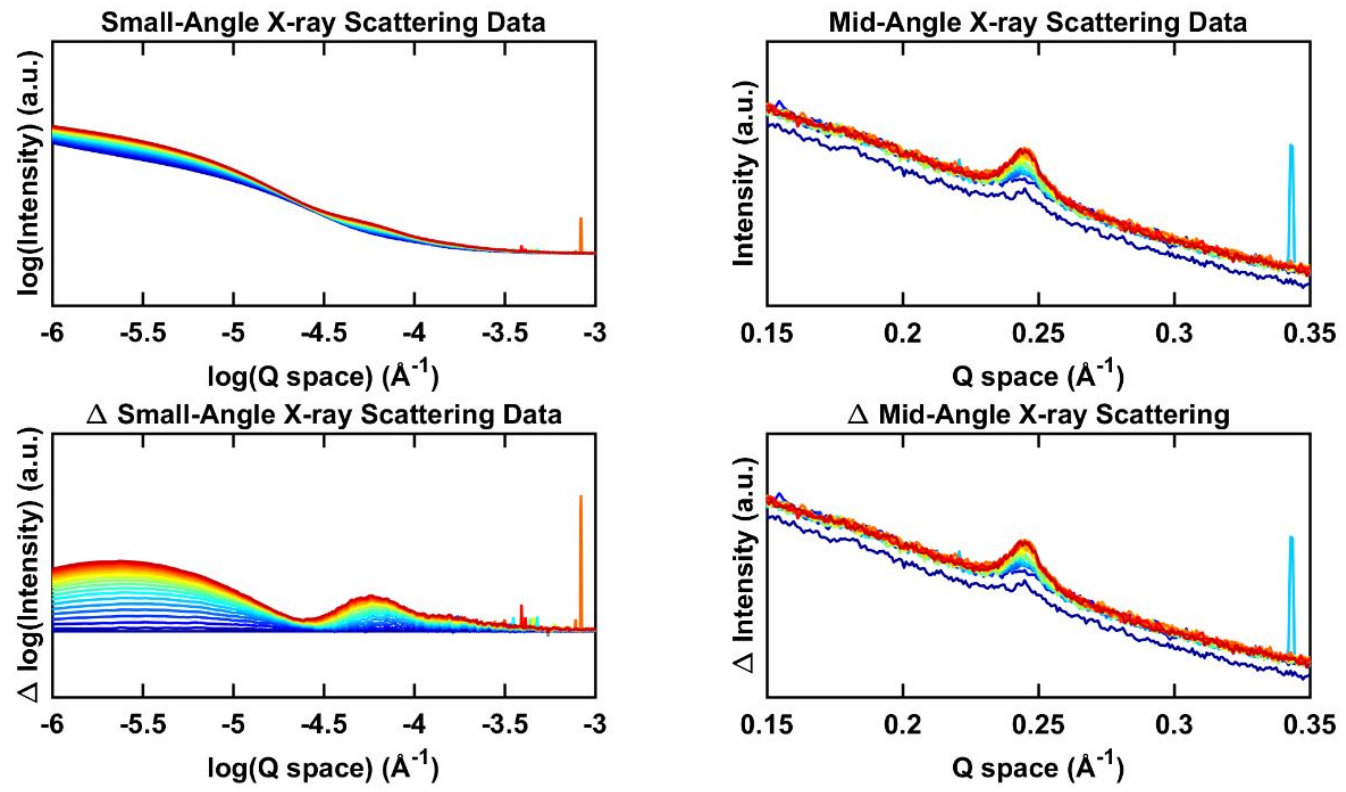

Figure S23. Growth Experiment [Monomer] $=0.23 \mathrm{mM}$.
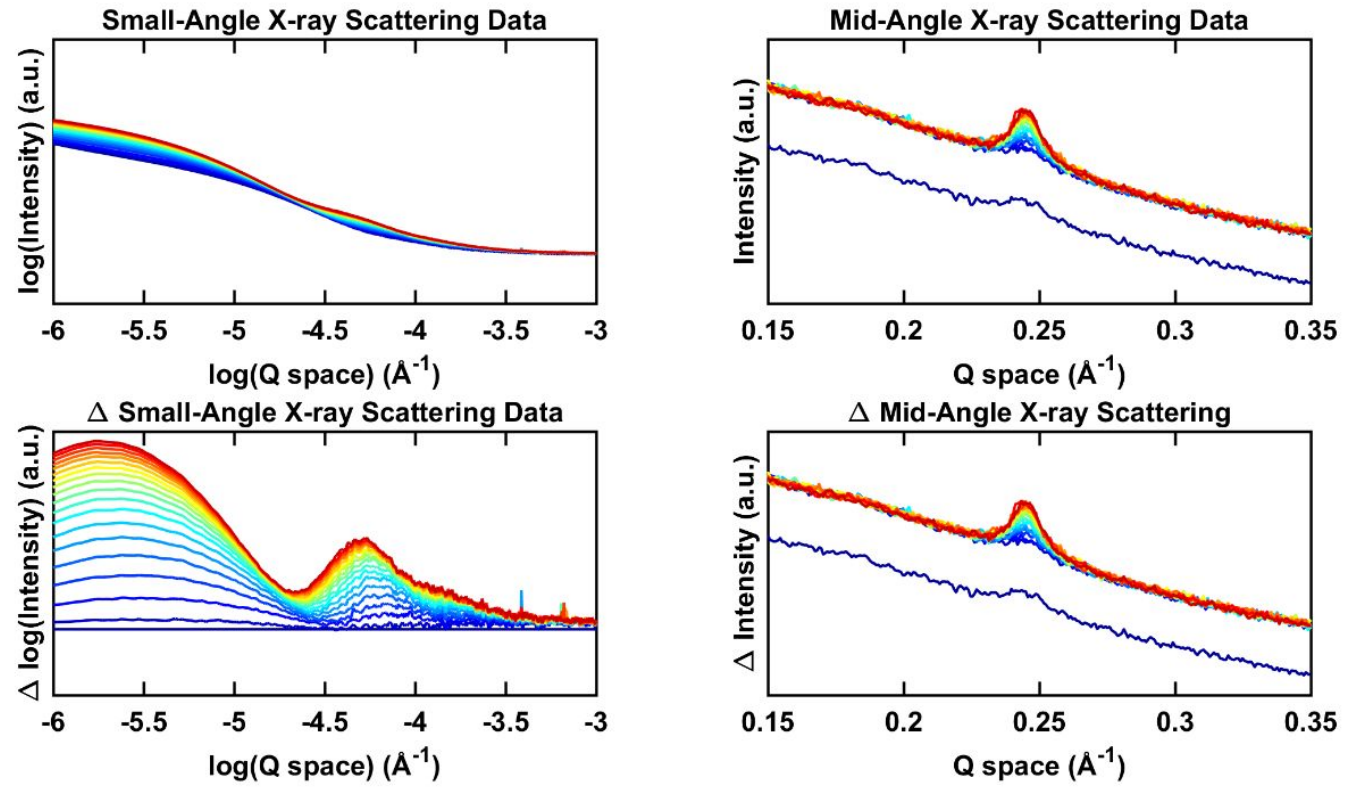

Figure S24. Growth Experiment [Monomer] $=0.28 \mathrm{mM}$. 

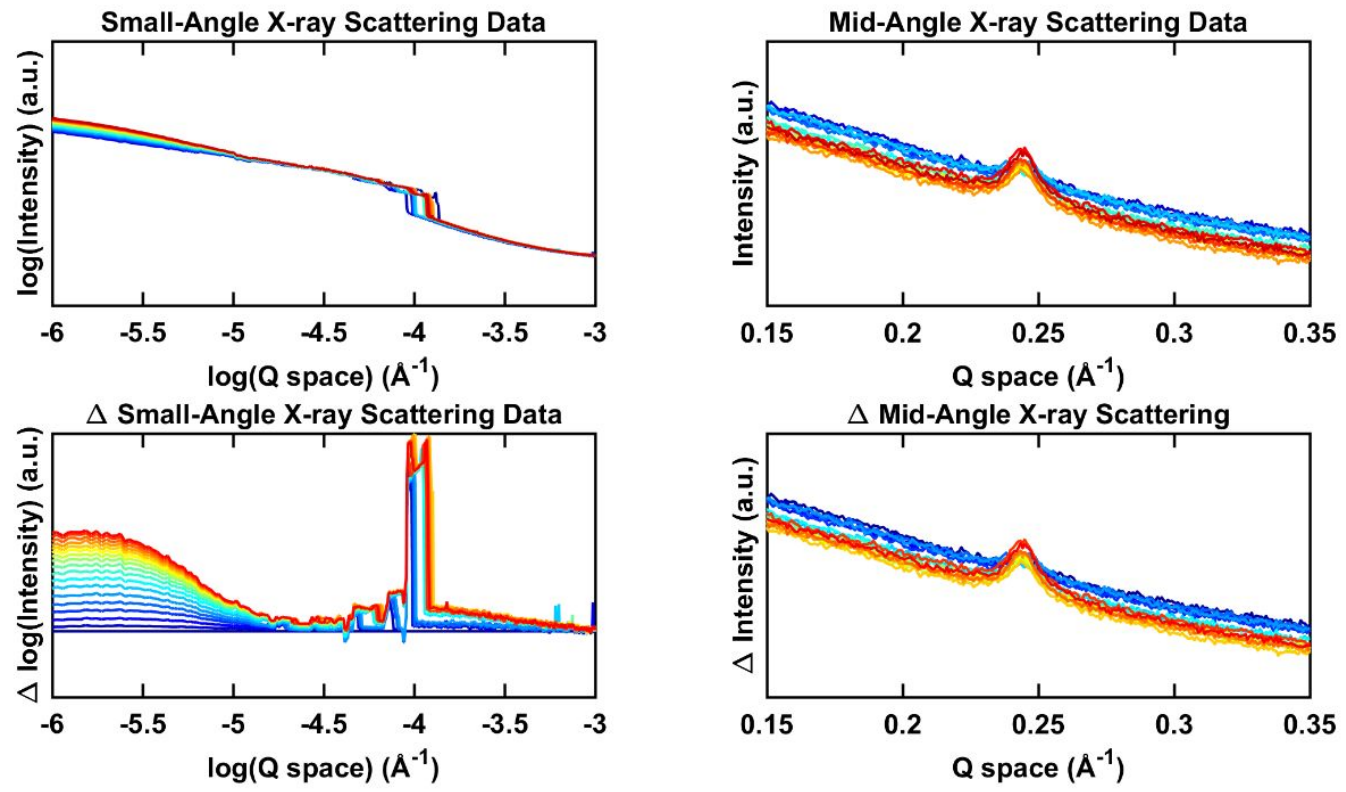

Figure S25. Growth Experiment [Monomer] $=0.33 \mathrm{mM}$.
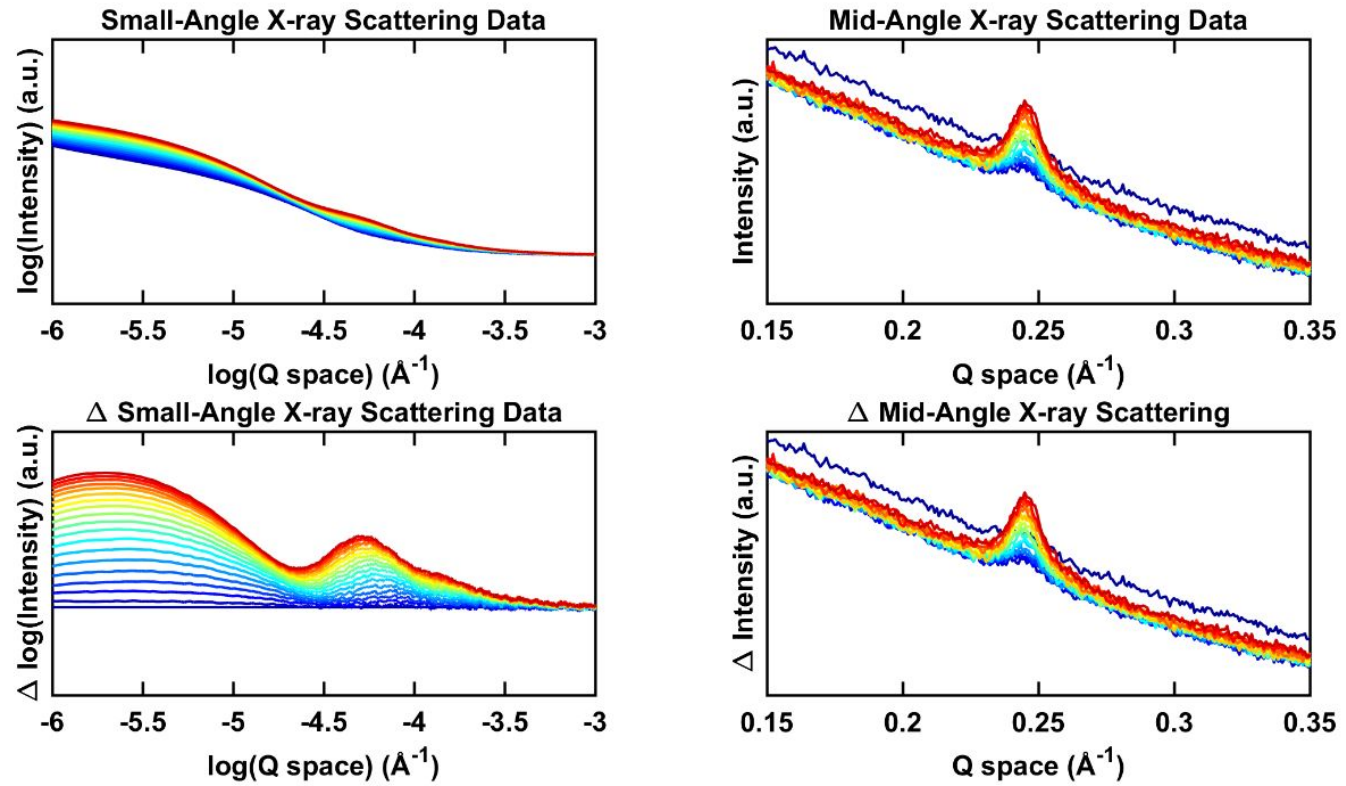

Figure S26. Growth Experiment [Monomer] $=0.38 \mathrm{mM}$. 

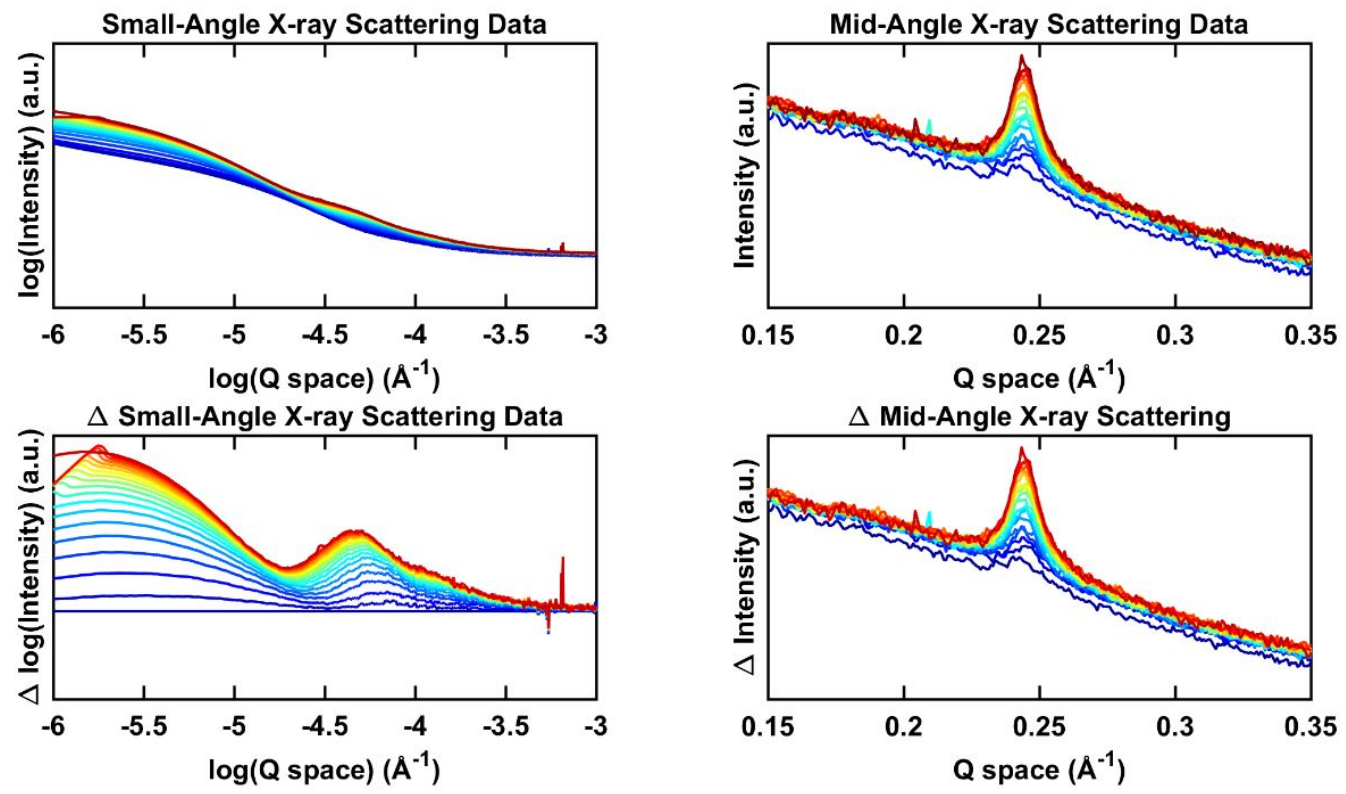

Figure S27. Growth Experiment [Monomer] $=0.48 \mathrm{mM}$. 


\section{In Situ X-Ray Scattering on Other Systems}
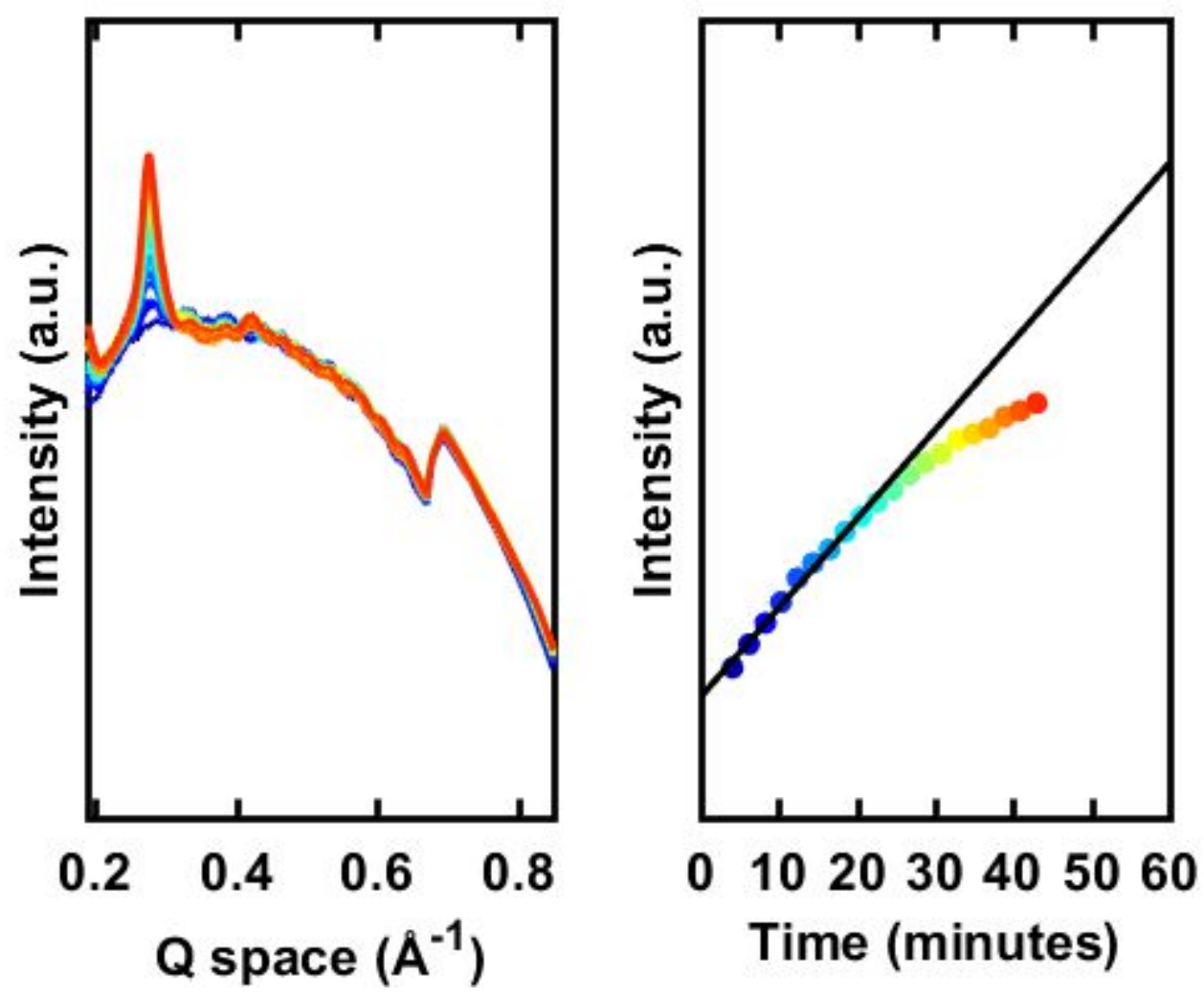

Figure S28. Nucleation Experiment on DPB-COF [ $4 \mathrm{mM}]$. DPB-COF is Formed from the Condensation of 4,4'-Diphenylbutadiynebis(boronic acid) and 2,3,6,7,10,11Hexahydroxytriphenylene. 

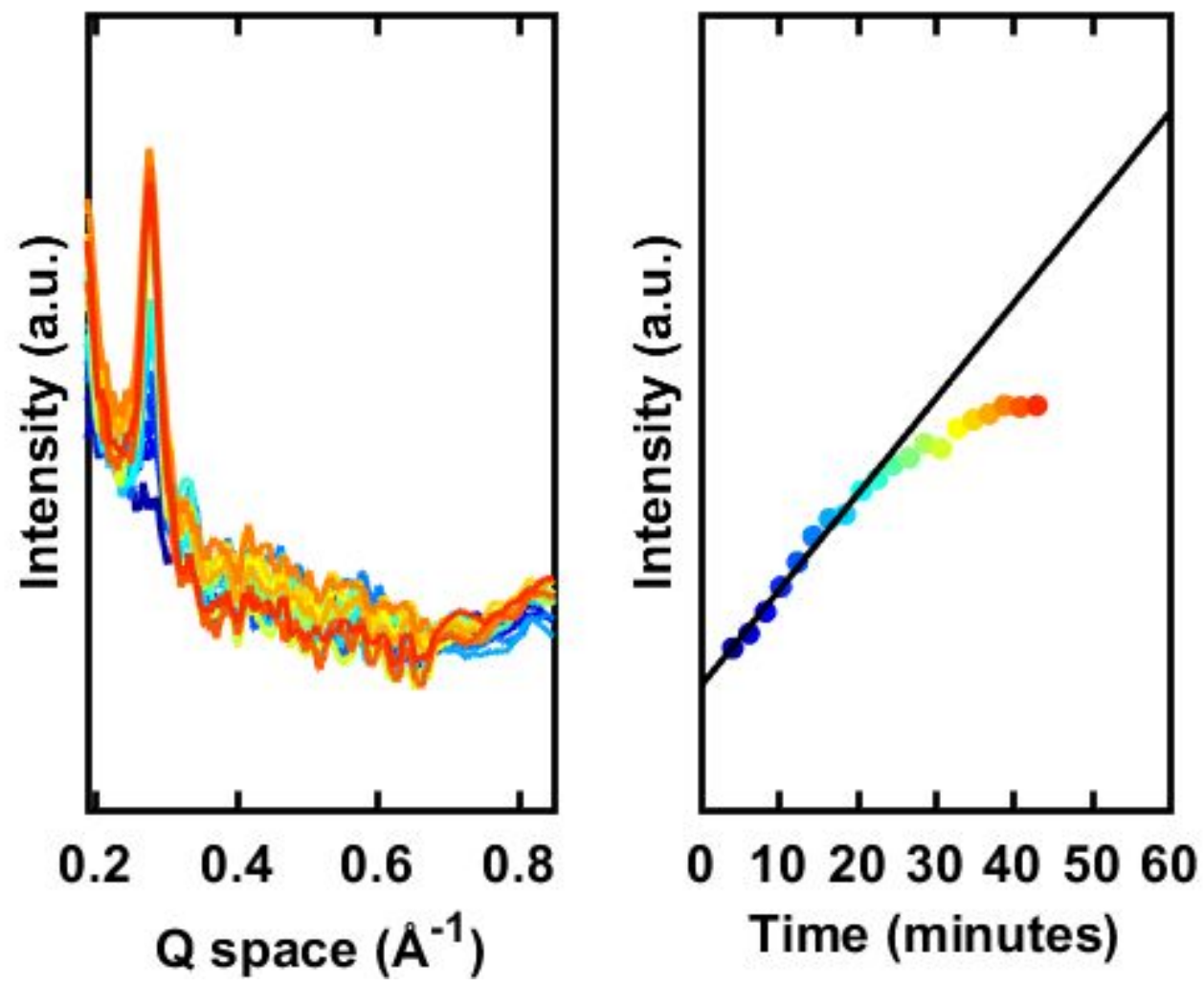

Figure S29. Nucleation Experiment on DPB-COF [2mM]. 

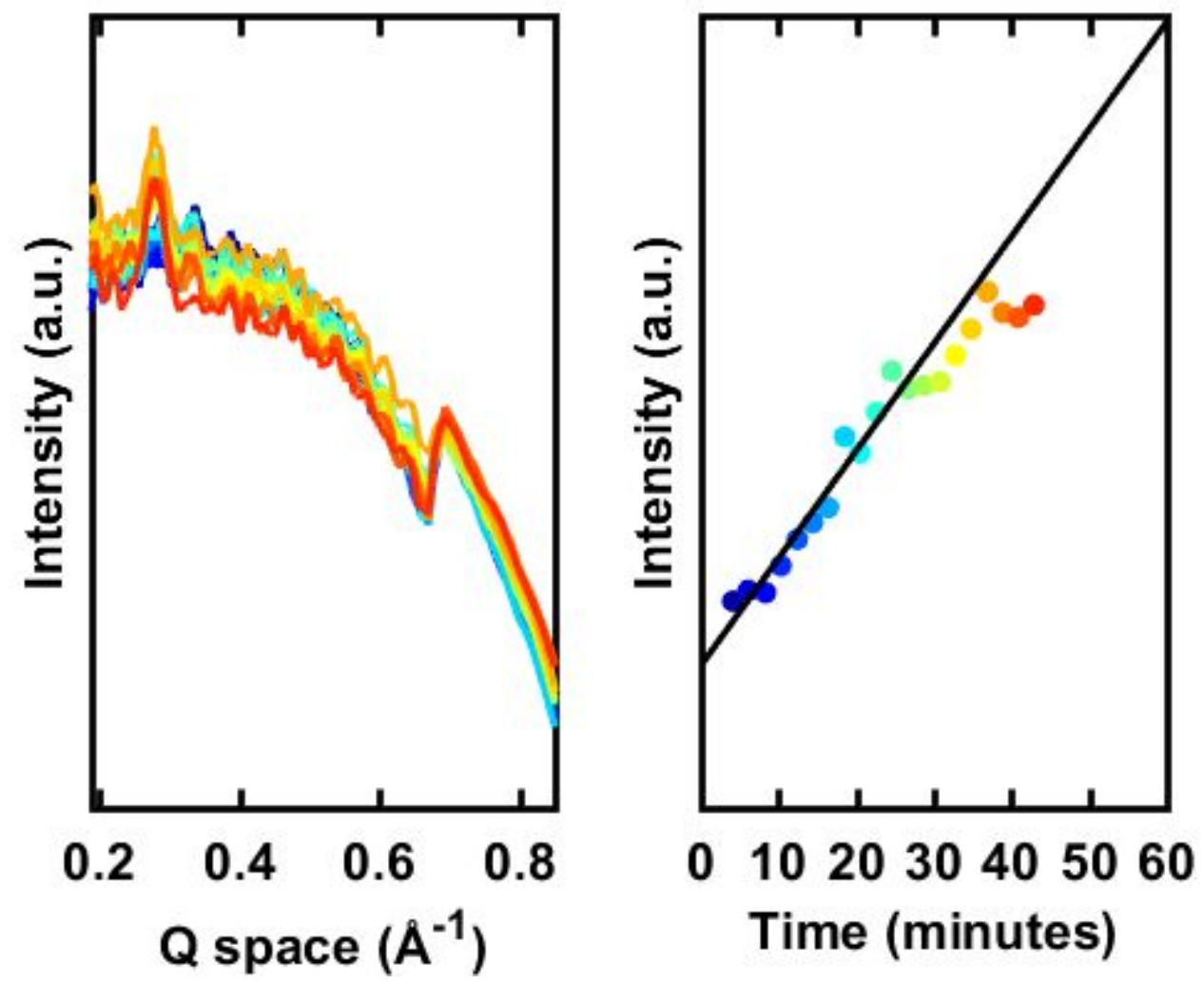

Figure S30. Nucleation Experiment on DPB-COF [1mM]. 


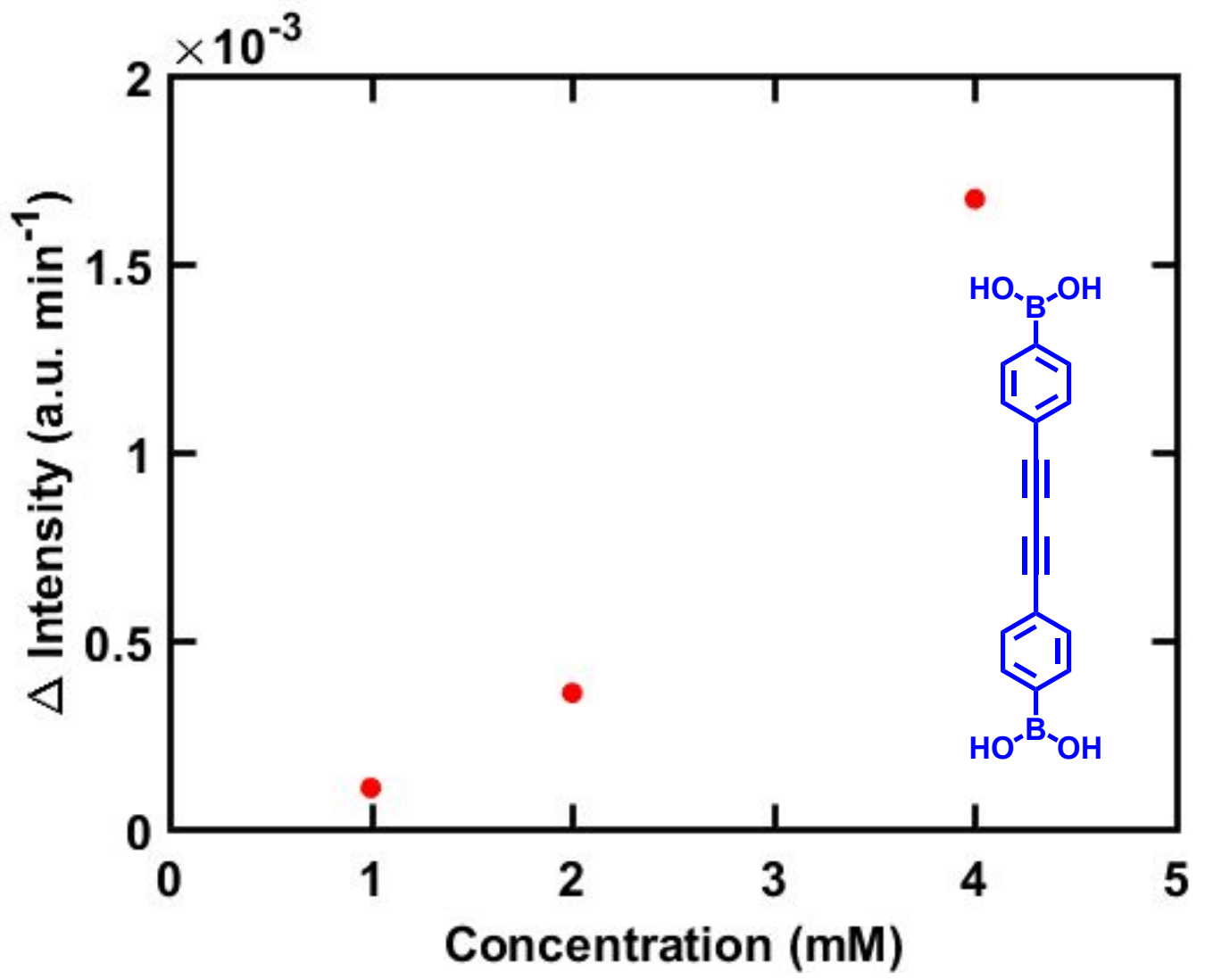

Figure S31. Rate of Intensity of the $<100>$ Bragg Feature as a Function of Initial Monomer Concentration for DPB-COF. DPB-COF is Formed from the Condensation of 4,4'Diphenylbutadiynebis(boronic acid) (inset) and 2,3,6,7,10,11-Hexahydroxytriphenylene. 

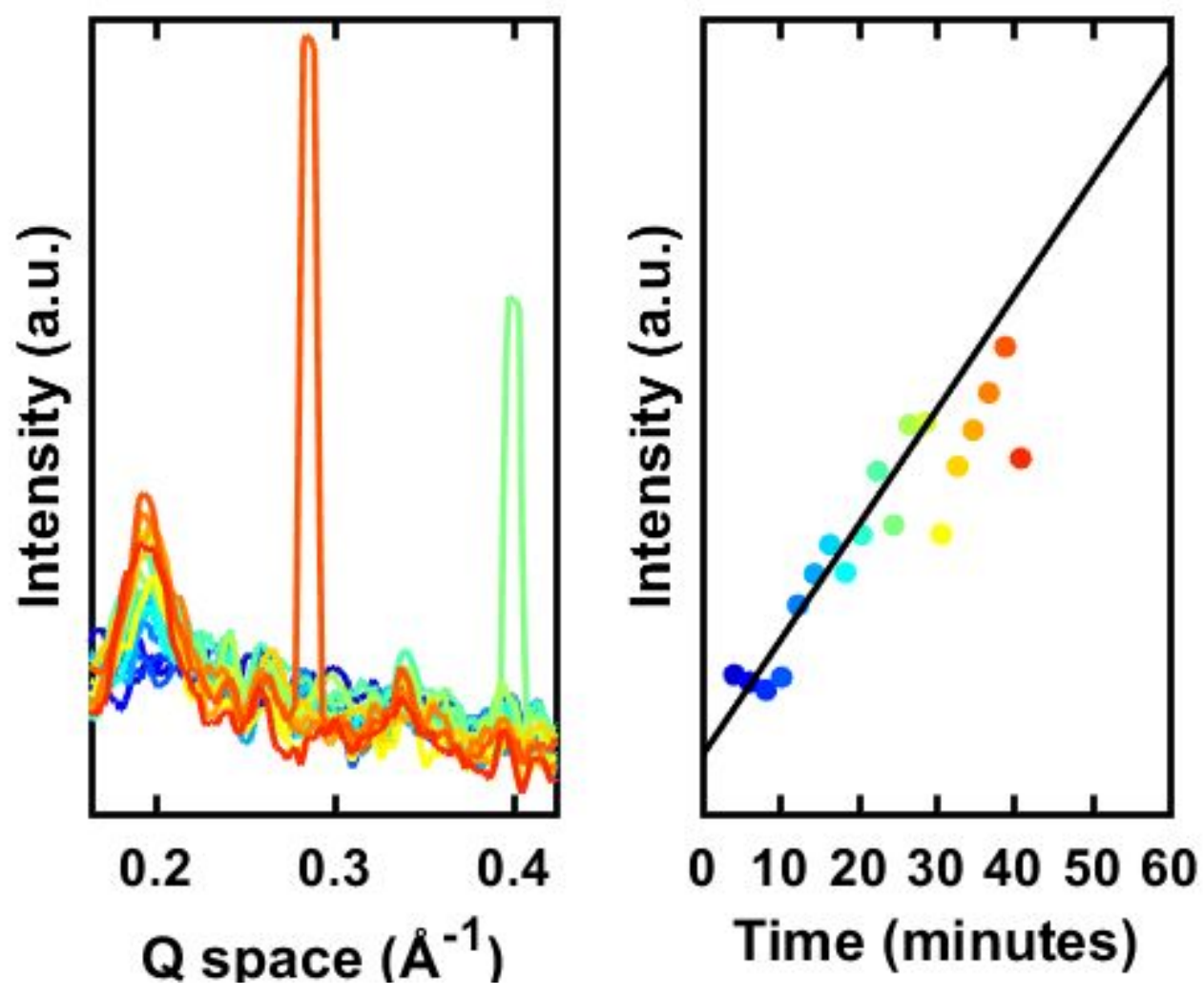

Figure S32. Nucleation Experiment on TP-COF [4mM]. TP-COF is Formed from the Condensation of 2,7-Pyrenebis(boronic acid) and 2,3,6,7,10,11-Hexahydroxytriphenylene. 

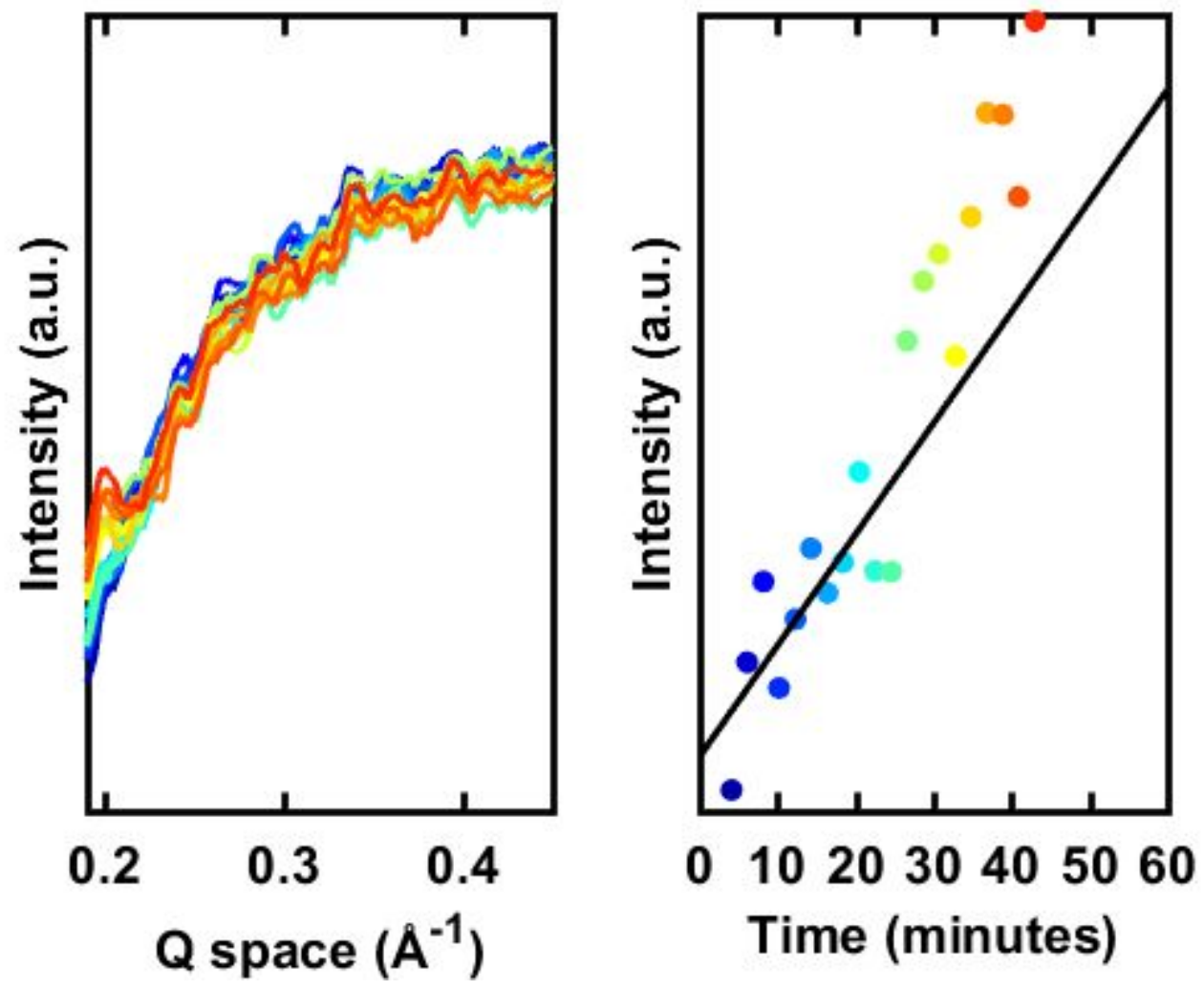

Figure S33. Nucleation Experiment on TP-COF [2mM]. 

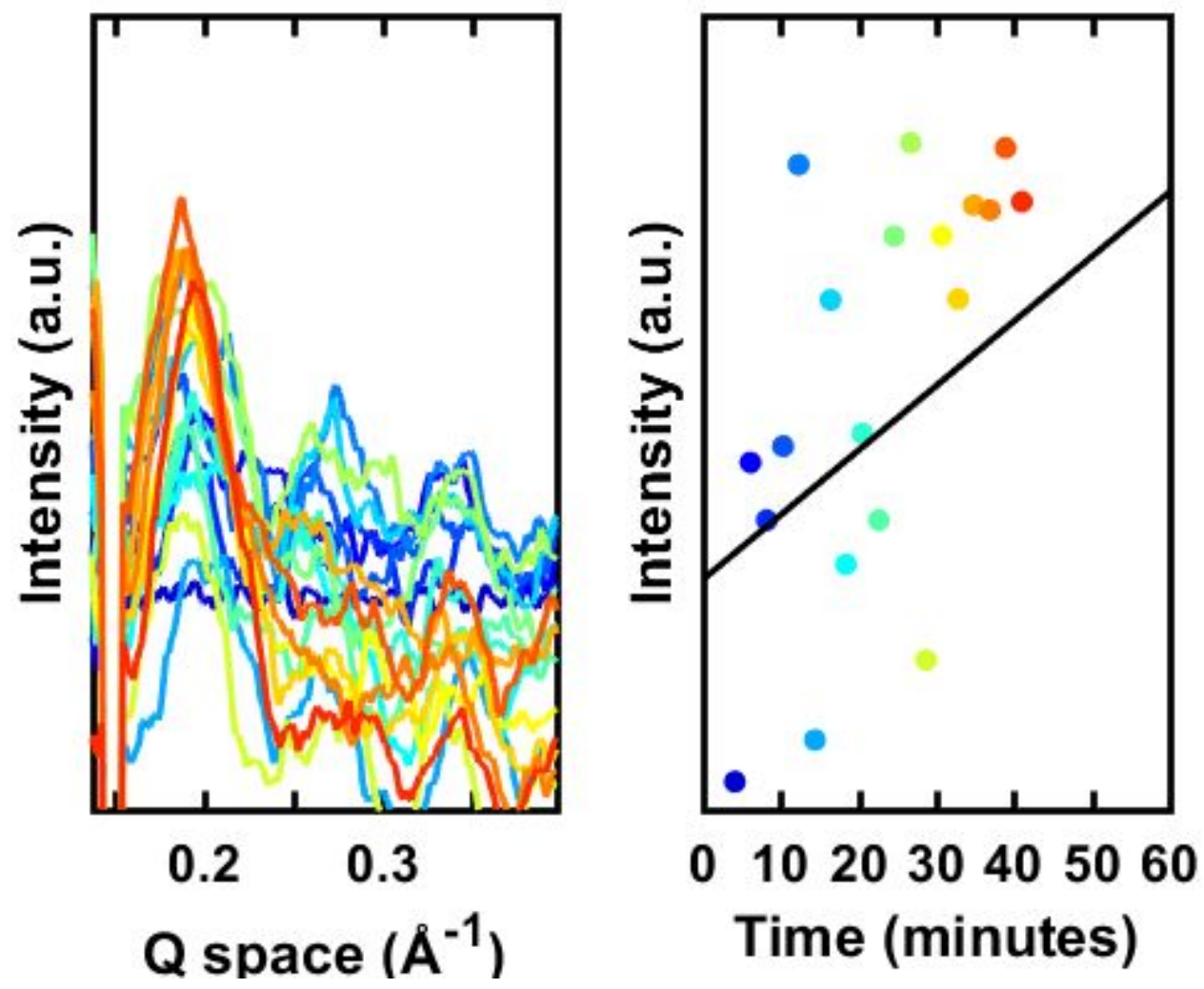

Figure S34. Nucleation Experiment on TP-COF [1mM]. 


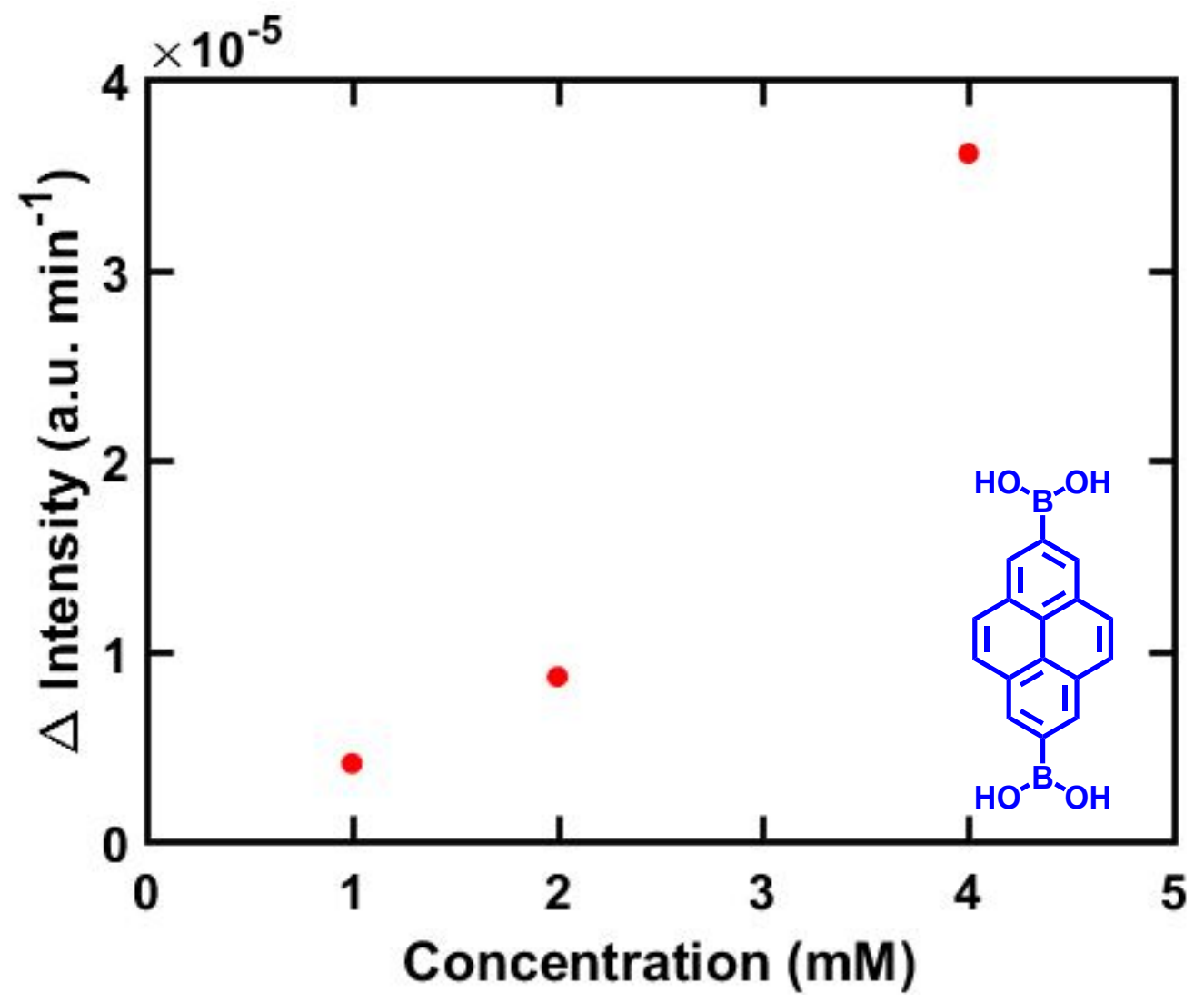

Figure S35. Rate of Intensity of the $<100>$ Bragg Feature as a Function of Initial Monomer Concentration for TP-COF. TP-COF is Formed from the Condensation of 2,7Pyrenebis(boronic acid) (inset) and 2,3,6,7,10,11-Hexahydroxytriphenylene. 


\section{Simulated X-ray Diffraction Models}

COF powders were collected by centrifugation of colloidal suspensions and were rinsed with blank solvent mixture to remove unreacted starting materials. Powder X-ray diffraction (PXRD) patterns were obtained at room temperature on a STOE-STADI P powder diffractometer equipped with an asymmetric curved Germanium monochromator. ( $\mathrm{Cu}-\mathrm{K} \alpha$ radiation, $\lambda=1.54056 \AA$ ) and onedimensional silicon strip detector (MYTHEN2 1K from DECTRIS). The line-focused Cu X-ray tube was operated at $40 \mathrm{kV}$ and $40 \mathrm{~mA}$. The as-obtained powder samples were sandwiched between two acetate foils (polymer sample with neither Bragg reflections nor broad peaks above $10^{\circ} 2 \theta$ ) mounted in flat plates with a disc opening diameter of $8 \mathrm{~mm}$ and measured in transmission geometry in a rotating holder. The patterns were recorded in the $2 \theta$ range of $0-36^{\circ}$ for an overall exposure time of $25 \mathrm{~min}$. The instrument was calibrated against a NIST Silicon standard (640d) prior to the measurement.

Crystal modeling of the COF structures was carried out using the Materials Studio (ver. 5.0) suite of programs by Accelrys. The initial structures were constructed piecewise starting with a primitive hexagonal unit cell with space group P6. The $a$ cell parameter was estimated according to the distance between the center of the vertices for each COF, and the $c$ parameter was arbitrarily chosen as $3.35 \AA$. The structures were optimized using the Geometry Optimization routine including energy minimization with cell parameters optimization, using the parameters from the Universal Force Field. Calculation of the simulated powder diffraction patterns and Pawley refinements were performed with the Materials Studio Reflex Plus Module using a BraggBrentano geometry. The observed diffraction patterns were subjected to a polynomial background subtraction and then to Pawley refinement wherein peak profiles were refined using the PseudoVoigt peak shape function and asymmetry was corrected using the Berar-Baldinozzi function. Crystallite size was then estimated by the LeBail method which was Pawley refined to the experimental data.

The Pawley refined structure was then partially offset and a simulated pattern of this structure was also generated. These simulated patterns strongly suggest that the COF-5 synthesized structure is entirely eclipsed.

CIF Files of the Pawley refined structure are included as supplemental attachments. 


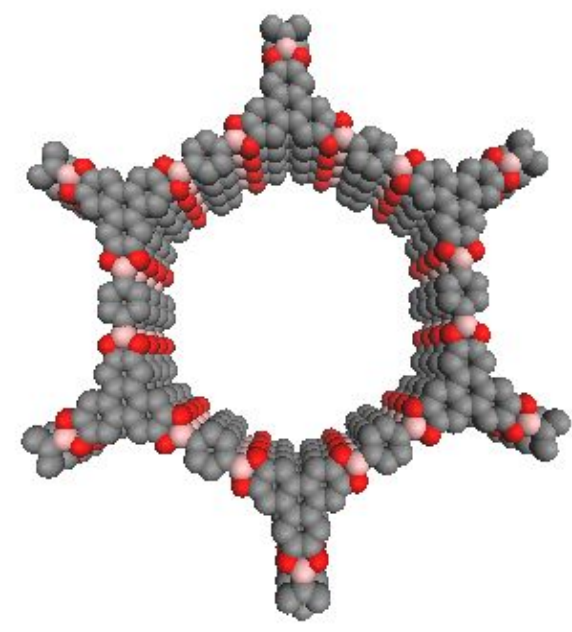

Figure S36. Eclipsed COF-5 Structure.

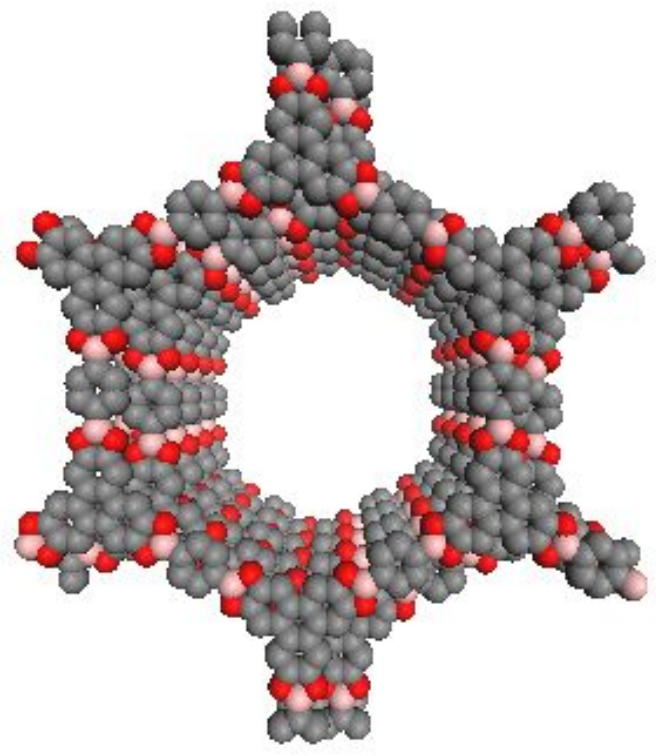

Figure S37. Offset COF-5 Structure. 


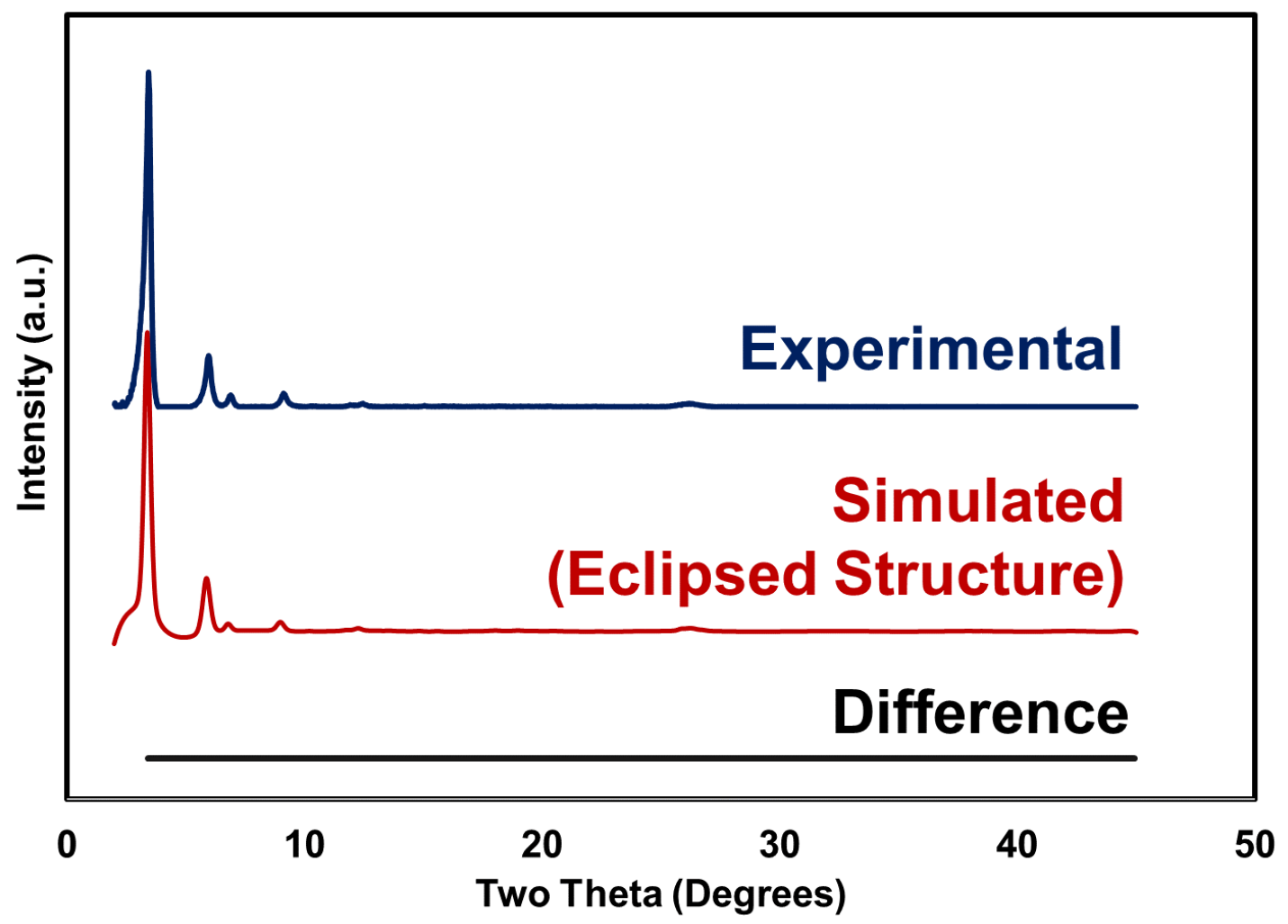

Figure S38. Comparison of the Experimental X-Ray Diffraction Patterns of COF-5 and Their Simulation Based on an Entirely Eclipsed Structure. The Simulated Data Include a LeBail Parameterization in the Pawley Refinement for Finite Grain Size Effects, Leading to Broadening of the Diffraction Features. 


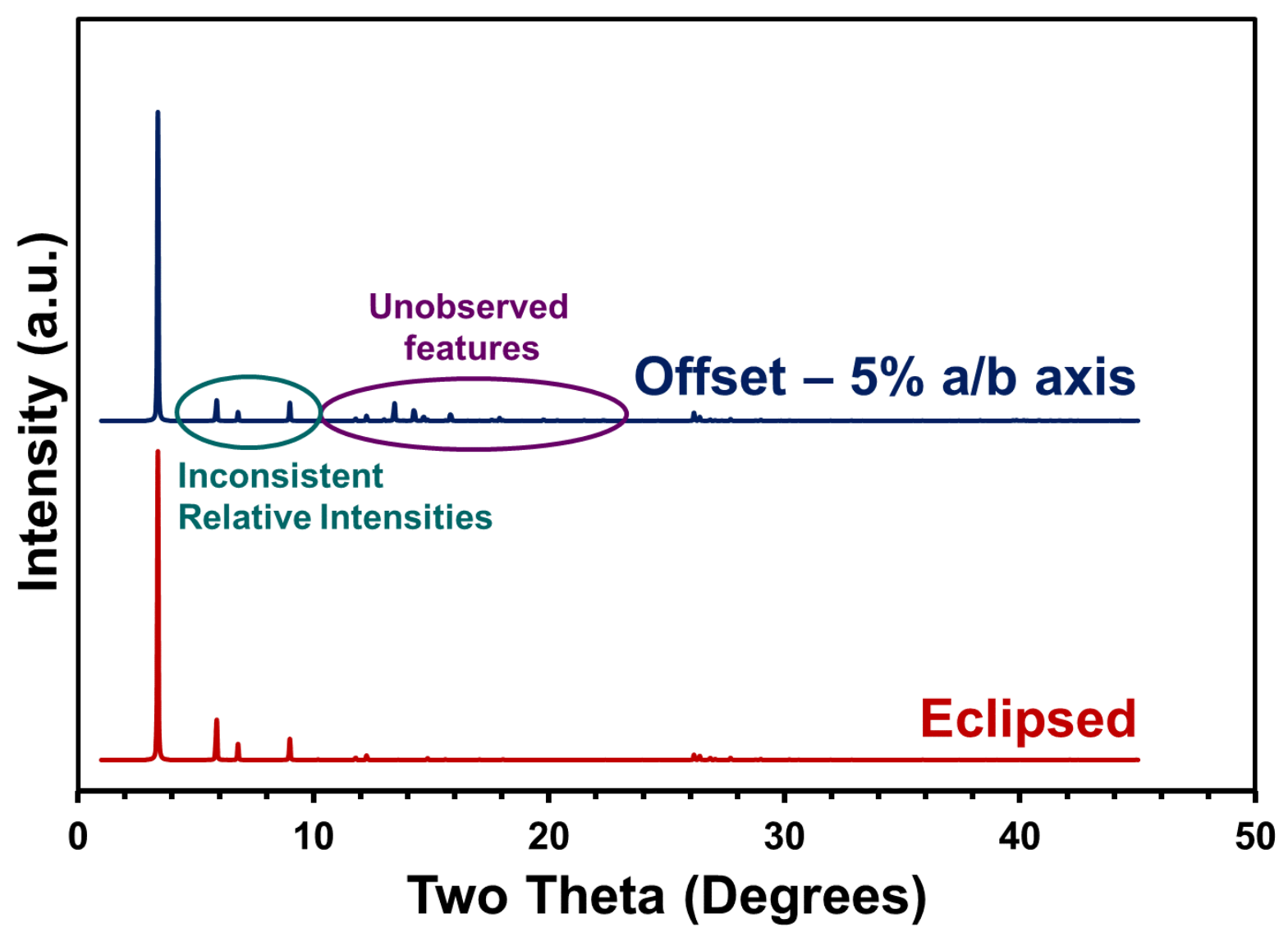

Figure S39. Comparison of the Simulated COF-5 X-Ray Diffraction Patterns Based on Eclipsed and Partially Offset Structures. Here, Infinite Grain Sizes Are Assumed. Green and Purple Circles Highlight the Peaks in the Partially Offset Structures That Are Inconsistent with the Experimental Data in Figure S38. 


\section{Derivation of Eqs. (4)-(8)}

The monomer consumption rate from nucleation is the product of the nucleation rate and the nucleus size:

$\omega_{\text {monomer }, n u c}=\mathrm{N}_{\mathrm{nuc}} J_{n u c}$

where $\mathrm{N}_{\text {nuc }}$ is the nucleus size (in number of HHTP units).

We substitute Eq. (1) in the main text into Eq. (S1):

$\omega_{\text {monomer }, \text { nuc }}=\mathrm{N}_{\text {nuc }} k_{\text {nucleation }} C_{\text {monomer }}^{2}$

Therefore, we have:

$\omega_{\text {monomer }, \text { nuc }} \propto C_{\text {monomer }}^{2}$

Eq. (S3) is Eq. (4) in the main text.

For simplicity, we assume that 2D COFs have a cylindrical shape with a fixed diameter to height ratio $\alpha(=d / h)$ during growth. The volume of a crystal $(V)$ is expressed as:

$V=\frac{\pi d^{2} h}{4}=\frac{\pi d^{3}}{4 \alpha}$

The volume change of a crystal is:

$\frac{d V}{d t}=\frac{d V}{d d} \frac{d d}{d t}$

Since $d d / d t$ is in-plane growth rate, we have:

$\frac{d V}{d t}=\frac{3 \pi d^{2} k_{\text {growth,in-plane }} C_{\text {monomer }}}{4 \alpha}$

The total volume change of all crystals equals:

$\frac{d V_{\text {tot }}}{d t}=\frac{3 \pi d^{2} k_{\text {growth,in-plane }} C_{\text {monomer }}}{4 \alpha} C_{\text {nuc }, 0} V_{o l} \mathrm{~N}_{\mathrm{A}}$ 
where $\mathrm{Vol}$ is the volume of the solution and $\mathrm{N}_{\mathrm{A}}$ is the Avogadro number. The total monomer number consumption is approximated to be proportional to the total volume change:

$$
\omega_{\text {monomer, growth }}=\frac{d V_{t o t}}{d t} \beta / \mathrm{N}_{\mathrm{A}} / V_{o l}
$$

where $\beta$ (in $\mathrm{nm}^{-3}$ ) is the number of monomer (HHTP) units per unit volume in the crystal.

Substituting Eq. (S7) into (S8), we have:

$$
\omega_{\text {monomer, growth }}=\frac{3 \pi d^{2} k_{\text {growth, in-plane }} C_{\text {nuc }, 0} \beta C_{\text {monomer }}}{4 \alpha}
$$

Therefore, we have:

$\omega_{\text {monomer, growth }} \propto d^{2} C_{\text {monomer }}$

Eq. (S10) is Eq. (5) in the main text.

The total monomer consumption from nucleation and growth can be obtained by summing Eqs. (S2) and (S9)

$\omega_{\text {consump }}=\frac{3 \pi d^{2} k_{\text {growth, in-plane }} C_{\text {monomer }}}{4 \alpha} C_{\text {nuc }, 0} \beta+\mathrm{N}_{\text {nuc }} k_{\text {nucleation }} C_{\text {monomer }}^{2}$

Since:

$d=k_{\text {growth,in-plane }} C_{\text {monomer }} t+d_{0}$

We have:

$\omega_{\text {consump }}=\frac{3 \pi k_{\text {growth, in }- \text { plane }} \beta C_{\text {nuc }, 0} C_{\text {monomer }}}{4 \alpha}\left(k_{\text {growth, in-plane }} C_{\text {monomer }} t+d_{0}\right)^{2}+\mathrm{N}_{\text {nuc }} k_{\text {nucleation }} C_{\text {monomer }}^{2}$

Eq. (S13) is Eq. (7) in the main text.

At a constant monomer concentration, the time for in-plane growth by $l$ is:

$$
t_{\text {growth }}=\frac{l}{v_{\text {in-plane }}}=\frac{l}{k_{\text {growth, in-plane }} C_{\text {monomer }}}
$$


The time for the production of new nuclei of concentration of $\gamma C_{n u c, 0}$ is:

$t_{n u c=} \frac{\gamma C_{n u c, 0}}{J_{n u c}}=\frac{\gamma C_{n u c, 0}}{k_{\text {nucleation }} C_{\text {monomer }}^{2}}$

Let $t_{\text {growth }}=t_{\text {nuc }}$, we have:

$C_{\text {monomer }}=\frac{\gamma C_{\text {nuc }, 0} k_{\text {growth }, \text { in }- \text { plane }}}{l k_{\text {nucleation }}}$

By definition, $C^{*}=C_{\text {monomer }}$. Therefore, we have:

$C^{*}=\frac{\gamma C_{n u c, 0} k_{\text {growth,in-plane }}}{l k_{\text {nucleation }}}$

Eq. (S17) is Eq. (6) in the main text.

Substituing Eq. (S17) into Eq. (S14), we have:

$$
t_{\text {growth }}=\frac{l}{k_{\text {growth,in-plane }} \frac{\gamma C_{\text {nuc }, 0} k_{\text {growth, }, \text { plane }}}{l k_{\text {nucleation }}}}=\frac{k_{\text {nucleation }} l^{2}}{k_{\text {growth,in-plane }} \gamma C_{\text {nuc }, 0} k_{\text {growth,in-plane }}}
$$

Since $\tau=t_{\text {growth }}$, we have:

$$
\tau=\frac{k_{\text {nucleation }} l^{2}}{k_{\text {growth, in-plane growth }}^{2} \gamma C_{\text {nuc }, 0}}
$$

Eq. (S19) is Eq. (8) in the main text.

\section{References}

(1) Li, H. Y.; Chavez, A. D.; Li, H. F.; Li, H.; Dichtel, W. R.; Brédas, J.-L., Nucleation and Growth of Covalent Organic Frameworks from Solution: The Example of COF-5, J. Am. Chem. Soc. 2017, 139, 16310.

(2) Evans, A. M.; Parent, L. R.; Flanders, N. C.; Bisbey, R. P.; Vitaku, E.; Kirschner, M. S.; Schaller, R. D.; Chen, L. X.; Gianneschi, N. C.; Dichtel, W. R., Seeded Growth of Single-Crystal Two-Dimensional Covalent Organic Frameworks, Science 2018, 361, 52. 